\title{
Shapley and Banzhaf values as probability transformations
}

\author{
ENRIQUE MIRANDA \\ Department of Statistics and O.R., University of Oviedo, C/Federico García Lorca, 18 \\ Oviedo, 33007 Spain \\ mirandaenrique@uniovi.es \\ IGNACIO MONTES \\ Department of Statistics and O.R., University of Oviedo, C/Federico García Lorca, 18 \\ Oviedo, 33007 Spain \\ imontes@uniovi.es \\ Received (received date) \\ Revised (revised date)
}

\begin{abstract}
We investigate the role of some game solutions, such the Shapley and the Banzhaf values, as probability transformations. The first one coincides with the pignistic transformation proposed in the Transferable Belief Model; the second one is not efficient in general, leading us to consider its normalized version. We study a number of particular models of lower probabilities: minitive measures, coherent lower probabilities, as well as the lower probabilities induced by comparative or distortion models. For them, we provide some alternative expressions of the Shapley and Banzhaf values and study under which conditions they belong to the core of the lower probability.
\end{abstract}

Keywords: Game solutions, coherent lower probabilities, belief functions, minitive measures, comparative models, core, Shapley value, Banzhaf value, pignistic transformation.

\section{Introduction}

As discussed in ${ }^{1}$, there are two main interpretations of set functions, or capacities: on the one hand, we can regard them as representations of uncertainty, where the capacity of a set $A$ measures our belief that the outcome of an experiment belongs to $A$. In this manner we can consider a number of models that generalize probability theory, and that are sometimes gathered together under the term imprecise probabilities. These include for instance Choquet capacities ${ }^{2}$, belief functions ${ }^{3}$, possibility measures ${ }^{4}$ or coherent lower probabilities ${ }^{5}$, among many others. One possible interpretation of these models is that of lower bounds of some precise, but unknown, probability distribution over the set of outcomes.

The second interpretation arises within cooperative game theory, and regards each subset $A$ of the possibility space as a set of players, and the capacity of $A$ as the payoff that is guaranteed for the coalition of the players in A. Although formally different, the theories stemming from the two approaches have a number of 
notions and results in common. For instance, the core of a capacity within imprecise probability theory is defined as the set of probability measures that dominate the capacity, and can be regarded as the set of probability models that are compatible with the available information. The exact same concept arises in cooperative game theory, with the interpretation of the set of individual payoffs that cannot be improved by any coalition.

One important problem within imprecise probabilities is that of eliciting a (precise) probability measure from an imprecise model. This is usually referred to as a probability transformation, and has been approached in many different ways: we can consider for instance the probability measure that minimizes (some) distance to the lower probability ${ }^{6}$, that with the maximum entropy ${ }^{7}$, etc. The problem has been considered with particular attention by the belief function community, where a number of different transformations have been proposed ${ }^{8,9}$. Among these, one of the most widely used is the so-called pignistic transformation, considered by Smets and proposed earlier by Dubois and Prade ${ }^{10}$ and Williams ${ }^{11}$.

It turns out that this transformation coincides with what Shapley proposed in 12 as a solution for a game, and that is understood as a distribution of the payoff among the players that satisfies a number of desirable properties. The link allows us to obtain the pignistic transformation as the center of gravity (a weighted average of the extreme points) of the set of probability measures associated with the nonadditive measure.

Inspired by this result, in this paper we investigate game solutions as probability transformations. On the one hand, we deepen in the properties of the Shapley value, studying if it can be expressed in terms of the extreme points in more general conditions than for 2-monotone lower probabilities, which is the result established by Shapley in ${ }^{13}$. In addition, we also study the role as a probability transformation of another popular solution proposed within game theory: the Banzhaf value.

One basic desirable property of a probability transformation of a lower probability is that it belongs to the core of this lower probability. This is essential if the latter is obtained as the envelope of a set of probability measures, as is the case when it is given an epistemic interpretation and also when we consider exact games in cooperative game theory, as we shall do in this paper. For this reason, we shall study among other things if we can guarantee the consistency of the Shapley and Banzhaf values with the lower probability it is induced from for different types of lower probabilities.

After introducing some preliminary concepts in Section 2, from Section 3 onwards we investigate the properties of the Shapley and Banzhaf values for some particular models: minitive measures (Section 3), 2-monotone capacities (Section 4) and coherent lower probabilities (Section 5). We conclude the paper in Section 6 with some additional remarks. 


\section{Preliminary concepts}

Let us recall some basic notions from the two theories we link in this paper: imprecise probabilities and cooperative game theory.

\subsection{Lower probabilities}

In this paper, we consider a finite possibility space $\Omega=\{1, \ldots, n\}$. A lower probability (also called capacity) on $\Omega$ is a function $\underline{P}: \mathcal{P}(\Omega) \rightarrow[0,1]$ that is monotone $(A \subseteq B \Rightarrow \underline{P}(A) \leq \underline{P}(B))$ and normalized $(\underline{P}(\emptyset)=0, \underline{P}(\Omega)=1)$. Its conjugate upper probability is given by $\bar{P}(A)=1-\underline{P}\left(A^{c}\right)$ for every $A \subseteq \Omega$, and its core $\mathcal{M}(\underline{P})$ is the set of additive models that are compatible with $\underline{P}$, in the sense that

$$
\mathcal{M}(\underline{P})=\{P: \mathcal{P}(\Omega) \rightarrow[0,1] \text { probability measure : } P(A) \geq \underline{P}(A) \quad \forall A \subseteq \Omega\} .
$$

We shall only consider in this paper lower probabilities $\underline{P}$ whose core is non-empty. These are said to avoid sure loss. They are called coherent if they are moreover the lower envelope of the core:

Definition 1. ${ }^{5}$ A lower probability $\underline{P}: \mathcal{P}(\Omega) \rightarrow[0,1]$ is coherent if and only if $\underline{P}(A)=\min \{P(A): P \in \mathcal{M}(\underline{P})\}$ for every $A \subseteq \Omega$.

One particular family of coherent lower probabilities are the 2-monotone ones ${ }^{2,14}$, which are those satisfying $\underline{P}(A \cup B)+\underline{P}(A \cap B) \geq \underline{P}(A)+\underline{P}(B)$ for any pair of subsets $A, B$ of $\Omega$. They are sometimes referred to as convex capacities.

The notion can be strengthened by considering complete monotonicity, which means that

$$
\underline{P}\left(\cup_{i=1}^{k} A_{i}\right) \geq \sum_{i=1}^{k} \underline{P}\left(A_{i}\right)-\sum_{i, j \in\{1, \ldots, k\}} \underline{P}\left(A_{i} \cap A_{j}\right)+\cdots+(-1)^{k+1} \underline{P}\left(\cap_{i=1}^{k} A_{i}\right)
$$

for every $k \in \mathbb{N}$ and every $A_{1}, \ldots, A_{k} \subseteq \Omega$.

Completely monotone lower probabilities are also called belief functions in the theory of evidence ${ }^{3}$. One of their advantages is that they are uniquely determined by their basic probability assignment $m: \mathcal{P}(\Omega) \rightarrow[0,1]$, which satisfies $\sum_{A \subseteq \Omega} m(A)=$ 1 , by means of the formula

$$
\underline{P}(A)=\sum_{B \subseteq A} m(B) .
$$

In order to determine the values of $\underline{P}(A)$ using Eq. (1), the only relevant terms in the summation are the focal sets, which are those sets $E$ such that $m(E)>0$.

More generally, any lower probability is determined by its Möbius inverse

$$
m(B)=\sum_{A \subseteq B}(-1)^{|B \backslash A|} \underline{P}(A),
$$

in the sense that this function $m$ determines $\underline{P}$ by means of Eq. (1); the only difference is that $m$ is non-negative if and only if $\underline{P}$ is a belief function. 


\subsection{Game solutions}

Within game theory, the possibility space $\Omega$ is interpreted as a set of players, and $\underline{P}(A)$ is then regarded as the gain that is guaranteed by the coalition of the players in $A$. Thus, a game is a function $\underline{P}: \mathcal{P}(\Omega) \rightarrow \mathbb{R}$ that provides the gain that is guaranteed to each coalition. In this paper, we focus on games taking values on $[0,1]$ and that are normalized and monotone; in other words, in those that can be regarded as lower probabilities.

Under the assumption of transferable utility, which means that all players have a common currency that is equally valued by all, the core of the game represents the set of distributions of the total payoff among the players (i.e., those probability distribution $P$ such that $\left.\sum_{i \in \Omega} P(\{i\})=1\right)$ that cannot be improved by any coalition (so that $P(A) \geq \underline{P}(A)$ for every coalition $A \subseteq \Omega$ ).

A solution of a game $\mathrm{e}^{\mathrm{a}}$ is a function that associates with each game a distribution of payoffs over the individual players in the game. Arguably the most important solution of a game is the so-called Shapley value ${ }^{12,13}$ :

Definition 2. ${ }^{12}$ Let $\underline{P}$ be a lower probability. Its Shapley value is given, for every player $i$, by

$$
\Phi(\underline{P})(i)=\sum_{A \nsupseteq\{i\}} \frac{t !(n-t-1) !}{n !}(\underline{P}(A \cup\{i\})-\underline{P}(A)),
$$

where $t=|A|$.

We shall also use $\Phi(\underline{P})$ to denote the probability measure determined by the above equation, so that $\Phi(\underline{P})(A):=\sum_{i \in A} \Phi(\underline{P})(i)$.

The Shapley value is the only solution of the game that satisfies the following four properties:

Efficiency $\sum_{i \in \Omega} \Phi(\underline{P})(i)=\underline{P}(\Omega)$.

Symmetry $\underline{P}(A \cup\{i\})=\underline{P}(A \cup\{j\}) \quad \forall A \subseteq \Omega \backslash\{i, j\} \Rightarrow \Phi(\underline{P})(i)=\Phi(\underline{P})(j)$.

Linearity $\Phi\left(\lambda_{1} \underline{P}_{1}+\lambda_{2} \underline{P}_{2}\right)=\lambda_{1} \Phi\left(\underline{P}_{1}\right)+\lambda_{2} \Phi\left(\underline{P}_{2}\right) \quad \forall \lambda_{1}, \lambda_{2} \in \mathbb{R}$ and for every pair of games $\underline{P}_{1}, \underline{P}_{2}$.

Null player $\underline{P}(A \cup\{i\})=\underline{P}(A) \quad \forall A \subseteq \Omega \Rightarrow \Phi(\underline{P})(i)=0$.

When the game $\underline{P}$ is 2-monotone, $\Phi(\underline{P})$ corresponds to the center of gravity of the core, and it can therefore be computed using the extreme points of $\mathcal{M}(\underline{P})$. From ${ }^{16}$, the extreme points of the core of a 2-monotone lower probability are related to the permutations of $\Omega$ : any permutation $\sigma$ defines an extreme point by means of the equation

$$
P_{\sigma}(\{\sigma(1), \ldots, \sigma(i)\}):=\underline{P}(\{\sigma(1), \ldots, \sigma(i)\}) \text { for } i=1, \ldots, n .
$$

\footnotetext{
aThe term solution is sometimes used within game theory to refer to a multifunction that to each game assigns a set of valid strategies, and what we use in this paper is then referred to as a solution concept.
} 
Proposition 1. ${ }^{12,13}$ Let $\underline{P}$ be a 2-monotone lower probability, and let $\Phi(\underline{P})$ denote its associated Shapley value. Then for every $i \in \Omega$ it holds that

$$
\Phi(\underline{P})(i)=\frac{\sum_{\sigma \in S^{\Omega}} P_{\sigma}(\{i\})}{n !},
$$

where $S^{\Omega}$ denotes the set of permutations of $\Omega$ and $P_{\sigma}$ is given by Eq. (4). As a consequence, $\Phi(\underline{P})$ belongs to the core $\mathcal{M}(\underline{P})$ of $\underline{P}$.

Note that in the above expression, if two different permutations $\sigma_{1} \neq \sigma_{2}$ give rise to the same extreme point $P_{\sigma}$, the latter is added twice; in other words, the Shapley value can be obtained as the weighted average of the extreme points, where the weight of an extreme point depends on the number of different permutations that induce it.

Interestingly, the Shapley value of a belief function coincides with what Smets called its pignistic transformation ${ }^{\mathrm{b}}$ within the Transferable Belief Model ${ }^{18}$, as shown in ${ }^{8}$. This means that we can also compute the Shapley value as:

$$
\Phi(\underline{P})(i)=\sum_{i \in B} \frac{m(B)}{|B|},
$$

where $m$ is determined by Eq. (2). In fact, the above formula can also be used for lower probabilities that are not belief functions, as shown in Section 3.6.1 in ${ }^{1}$. As a consequence it can be used to justify the use of the pignistic transformation beyond this framework. See ${ }^{19,20}$ for some works making use of the pignistic transformation.

Another popular solution of a game is the so-called Banzhaf value $\left({ }^{21}\right.$; see also $\left.{ }^{15}\right)$, which is often used as a power index in voting systems.

Definition 3. ${ }^{21}$ Let $\underline{P}$ be a lower probability. Its Banzhaf value is given by

$$
B(\underline{P})(i)=\frac{1}{2^{n-1}} \sum_{A \nsupseteq\{i\}}(\underline{P}(A \cup\{i\})-\underline{P}(A)) \text { for every } i \in \Omega .
$$

It satisfies the symmetry, linearity and null player axioms ${ }^{22}$. However, and unlike the Shapley value, the equation above does not produce a probability mass function, because it does not satisfy the efficiency axiom: we may have $\sum_{i \in \Omega} B(\underline{P})(i)>1$ or $\sum_{i \in \Omega} B(\underline{P})(i)<1$ (see the forthcoming Example 2). For this reason, it has been suggested ${ }^{23}$ to consider instead the normalized Banzhaf value, which is given by

$$
\Psi(\underline{P})(i)=\frac{B(\underline{P})(i)}{\sum_{j \in \Omega} B(\underline{P})(j)},
$$

and we shall denote $\Psi(\underline{P})(A)=\sum_{i \in A} \Psi(\underline{P})(i)$.

Although the normalized Banzhaf value does not share all the properties of the Banzhaf value ${ }^{24}$ (it loses for instance the linearity axiom), it has been axiomatized from the point of view of game theory in ${ }^{23}$.

$\overline{\mathrm{b} \text { See }}{ }^{11,17}$ for earlier proposals of this transformation. 
Remark 1. Both Shapley and Banzhaf values constitute examples of probabilistic values for games, using the terminology of Weber ${ }^{15}$. Given a game $\underline{P}$, a probabilistic value $S(\underline{P})$ would be determined by the formula

$$
S(\underline{P})(i)=\sum_{A \nsupseteq\{i\}} p_{A}^{i}(\underline{P}(A \cup\{i\})-\underline{P}(A)),
$$

where, for each player $i,\left\{p_{A}^{i}: A \nsupseteq\{i\}\right\}$ is a probability distribution over those coalitions that do not include $i$.

If we regard $\underline{P}(A \cup\{i\})-\underline{P}(A)$ as the reward for player $i$ for joining coalition $A$, then the probabilistic value $S(\underline{P})$ on $i$ could be interpreted as the expected reward for player $i$, considering his probabilities of joining the different coalitions. Both Shapley and Banzhaf values can be understood as probabilistic values:

- The Shapley value corresponds to the case $p_{A}^{i}=\frac{t !(n-t-1) !}{n !}$ for all $i$, where $t=|A|$ : the probability of joining a coalition depends on the size of the coalition, and all coalitions of the same size are equally likely;

- The Banzhaf values corresponds to $p_{A}^{i}=\frac{1}{2^{n-1}}$ : all coalitions are equally likely, irrespective of their size.

In this paper, we investigate the properties of the Shapley value and the normalized Banzhaf value as probability transformations of the core. Specifically, we shall study for which types of lower probabilities they are guaranteed to belong to their core, as well as provide some simpler expressions for a number of particular cases.

\section{Minitive measures}

We begin by considering a particular case of belief functions: minitive measures.

Definition 4. A lower probability $\underline{P}: \mathcal{P}(\Omega) \rightarrow[0,1]$ is called minitive when it satisfies.

$$
\underline{P}(A \cap B)=\min \{\underline{P}(A), \underline{P}(B)\} \quad \forall A, B \subseteq \Omega .
$$

In such case, its conjugate upper probability, given by $\bar{P}(A)=1-\underline{P}\left(A^{c}\right)$, is called maxitive and it satisfies $\bar{P}(A \cup B)=\max \{\bar{P}(A), \bar{P}(B)\}$ for every $A, B \subseteq \Omega$.

Since we are dealing with finite possibility spaces, minitive and maxitive measures are equivalent to necessity and possibility measures, respectively. Moreover, given conjugate minitive and maxitive measures $\underline{P}, \bar{P}$ on $\{1, \ldots, n\}$, it holds that $\max _{j=1, \ldots, n} \bar{P}(\{j\})=1$. Without loss of generality, we can assume that the elements in $\Omega$ are ordered so that $1=\bar{P}(\{1\}) \geq \bar{P}(\{2\}) \geq \ldots \geq \bar{P}(\{n\}) \geq 0$.

As particular cases, minitive measures include the unanimity games we shall mention later on; from the point of view of coalitional game theory, they correspond to games that are uniquely determined by the maximum rewards associated with the different players and the total order these maxima determine. 
It was proven by Nguyen ${ }^{25}$ that any minitive measure is in particular completely monotone, and therefore also 2-monotone. As a consequence, its Shapley value can also be obtained in this case as the center of gravity of the elements of the core (see Proposition 1). Furthermore, its focal sets are nested. Therefore, if $\underline{P}$ is a minitive measure, we can assume without loss of generality that its focal elements are among the sets $\{1, \ldots, j\}$ for $j=1, \ldots, n$.

Using the expression of the Shapley value in terms of the pignistic transformation, Dubois and Prade established the following formula:

Proposition 2. ${ }^{17,26}$ Let $\underline{P}$ be a minitive measure with conjugate maxitive measure $\bar{P}$. Then its Shapley value is given by:

$$
\Phi(\underline{P})(i)=\sum_{j=i}^{n} \frac{\bar{P}(\{j\})-\bar{P}(\{j+1\})}{j} \quad \forall i=1, \ldots, n,
$$

where we adopt the convention $\bar{P}(\{n+1\})=0$.

Using this result, if $m$ denotes the basic probability assignment associated with $\underline{P}$, the Shapley value can equivalently be expressed by:

$$
\Phi(\underline{P})(i)=\sum_{j=i}^{n} \frac{m(\{1, \ldots, j\})}{j} \quad \forall i=1, \ldots, n .
$$

This can also be derived using Eq. (6).

With respect to the normalized Banzhaf value, we have the following:

Proposition 3. Let $\underline{P}$ be a minitive measure, and denote by $m$ its basic probability assignment. Then its Banzhaf value is given by:

$$
B(\underline{P})(i)=\frac{1}{2^{n-1}} \sum_{j=i}^{n} 2^{n-j} m(\{1, \ldots, j\}) \quad \forall i=1, \ldots, n .
$$

Proof. Remember that we are assuming without loss of generality that $1=$ $\bar{P}(\{1\}) \geq \ldots \geq \bar{P}(\{n\})$, hence the focal sets associated with $\underline{P}$ are among the sets $\{1, \ldots, j\}$ for $j=1, \ldots, n$. Hence, $\underline{P}$ can be expressed by:

$$
\underline{P}(A)=\sum_{\{1, \ldots, j\} \subseteq A} m(\{1, \ldots, j\}) \quad \forall A \subseteq \Omega .
$$

Recall that the Banzhaf value is given by Eq. (7). However, note that if $\{1, \ldots, i-$ $1\} \nsubseteq A$, then the value $k=\max \{l:\{1, \ldots, l\} \subseteq A\}$ satisfies $k<i-1$. Therefore, it holds that:

$$
\begin{aligned}
\underline{P}(A \cup\{i\})-\underline{P}(A) & =\sum_{\{1, \ldots, j\} \subseteq A \cup\{i\}} m(\{1, \ldots, j\})-\sum_{\{1, \ldots, j\} \subseteq A} m(\{1, \ldots, j\}) \\
& =\sum_{j=1}^{k} m(\{1, \ldots, j\})-\sum_{j=1}^{k} m(\{1, \ldots, j\})=0 .
\end{aligned}
$$


Therefore, Eq. (7) can be simplified to:

$$
B(\underline{P})(i)=\frac{1}{2^{n-1}} \sum_{A \subseteq\{i+1, \ldots, n\}}(\underline{P}(A \cup\{1, \ldots, i\})-\underline{P}(A \cup\{1, \ldots, i-1\})) .
$$

Recall also that for any $A \subseteq\{i+1, \ldots, n\}$, if we denote $k=\max \{l:\{1, \ldots, l\} \subseteq$ $A \cup\{1, \ldots, i\}\} \geq i$, it holds that:

$$
\begin{aligned}
& \underline{P}(A \cup\{1, \ldots, i\})-\underline{P}(A \cup\{1, \ldots, i-1\}) \\
& \quad=\left(\sum_{j=1}^{k} m(\{1, \ldots, j\})\right)-\left(\sum_{j=1}^{i-1} m(\{1, \ldots, j\})\right)=\sum_{j=i}^{k} m(\{1, \ldots, j\}) .
\end{aligned}
$$

Thus, the only values that appear in the sum of the Banzhaf value are $m(\{1, \ldots, j\})$ for $j \geq i$. To see how many times any value $m(\{1, \ldots, j\}$ ) (for $j \geq i$ ) appears, note that, given any fixed $j \geq i$, it holds that:

- There is $\left(\begin{array}{c}n-j \\ 0\end{array}\right)=1$ set $A \cup\{1, \ldots, i\}$ with cardinality $j$ (the set $\{1, \ldots, j\}$ ) such that $\{1, \ldots, j\} \subseteq A \cup\{1, \ldots, i\}$.

- There are $\left(\begin{array}{c}n-j \\ 1\end{array}\right)$ sets $A \cup\{1, \ldots, i\}$ with cardinality $j+1$ such that $\{1, \ldots, j\} \subseteq A \cup\{1, \ldots, i\}$.

- ...

- There are $\left(\begin{array}{c}n-j \\ k\end{array}\right)$ sets $A \cup\{1, \ldots, i\}$ with cardinality $j+k$ such that $\{1, \ldots, j\} \subseteq A \cup\{1, \ldots, i\}$.

- ...

- There is $\left(\begin{array}{l}n-j \\ n-j\end{array}\right)=1$ set $A \cup\{1, \ldots, i\}$ with cardinality $n=j+n-j$ (the set $\{1, \ldots, n\})$ such that $\{1, \ldots, j\} \subseteq A \cup\{1, \ldots, i\}$.

Therefore, the value $m(\{1, \ldots, j\})$ appears $2^{n-j}$ times:

$$
\left(\begin{array}{c}
n-j \\
0
\end{array}\right)+\left(\begin{array}{c}
n-j \\
1
\end{array}\right)+\ldots+\left(\begin{array}{c}
n-j \\
n-j
\end{array}\right)=\sum_{k=0}^{n-j}\left(\begin{array}{c}
n-j \\
k
\end{array}\right)=2^{n-j}
$$

and as a consequence:

$$
B(\underline{P})(i)=\frac{1}{2^{n-1}} \sum_{j=i}^{n} 2^{n-j} m(\{1, \ldots, j\}) .
$$

This completes the proof.

From this result, it follows immediately the expression of the normalized Banzhaf value for minitive measures:

Corollary 1. Let $\underline{P}$ be a minitive measure, and denote by $m$ its basic probability assignment. Then its normalized Banzhaf value is given by:

$$
\Psi(\underline{P})(i)=\frac{\sum_{j=i}^{n} 2^{n-j} m(\{1, \ldots, j\})}{\sum_{j=1}^{n} j \cdot 2^{n-j} m(\{1, \ldots, j\})} \quad \forall i=1, \ldots, n .
$$


Proof. First of all, let us compute the sum of all the Banzhaf values:

$$
\sum_{i=1}^{n} B(\underline{P})(i)=\frac{1}{2^{n-1}} \sum_{i=1}^{n} \sum_{j=i}^{n} 2^{n-j} m(\{1, \ldots, j\}) .
$$

Now, the value $2^{n-j} m(\{1, \ldots, j\})$ appears in the previous sum for $i=1, \ldots, j$, whence Eq. (10) becomes:

$$
\sum_{i=1}^{n} B(\underline{P})(i)=\frac{1}{2^{n-1}} \sum_{j=1}^{n} j \cdot 2^{n-j} m(\{1, \ldots, j\}),
$$

and applying Eq. (8) we conclude that the normalized Banzhaf value is given by Eq. (9).

This result allows us to establish the following:

Proposition 4. Let $\underline{P}$ be a minitive measure. Then its normalized Banzhaf value $\Psi(\underline{P})$ belongs to its core $\mathcal{M}(\underline{P})$.

Proof. Let us show that $\Psi(\underline{P})(\{1, \ldots, k\}) \geq \underline{P}(\{1, \ldots, k\})$ for any $k=1, \ldots, n$. For simplicity, let $B=\{1, \ldots, k\}$. It follows from Eq. (9) that

$$
\begin{aligned}
\Psi(\underline{P})(B) & =\sum_{i=1}^{k} \Psi(\underline{P})(i)=\sum_{i=1}^{k} \frac{\sum_{j=i}^{n} 2^{n-j} m(\{1, \ldots, j\})}{\sum_{j=1}^{n} j \cdot 2^{n-j} m(\{1, \ldots, j\})} \\
& =\frac{\sum_{j=1}^{k} j \cdot 2^{n-j} m(\{1, \ldots, j\})}{\sum_{j=1}^{n} j \cdot 2^{n-j} m(\{1, \ldots, j\})} \\
& =\frac{\sum_{j=1}^{k} j \cdot 2^{n-j} m(\{1, \ldots, j\})}{\sum_{j=1}^{k} j \cdot 2^{n-j} m(\{1, \ldots, j\})+\sum_{j=k+1}^{n} j \cdot 2^{n-j} m(\{1, \ldots, j\})} \\
& \geq \frac{k \cdot 2^{n-k} \underline{P}(B)}{k \cdot 2^{n-k} \underline{P}(B)+(k+1) 2^{n-k-1}(1-\underline{P}(B))} .
\end{aligned}
$$

To see this inequality, note that a ratio $\frac{a}{a+b}$, where $a, b$ are non-negative numbers satisfying some constraints independent of each other, is minimized when we minimize $a$ and maximize $b$. Also, since $f(j)=j \cdot 2^{n-j}$ is a non-increasing function it holds that:

$$
\sum_{j=1}^{k} j \cdot 2^{n-j} m(\{1, \ldots, j\}) \geq k \cdot 2^{n-k} \sum_{j=1}^{k} m(\{1, \ldots, j\})=k \cdot 2^{n-k} \underline{P}(B),
$$

and

$$
\begin{aligned}
\sum_{j=k+1}^{n} j \cdot 2^{n-j} m(\{1, \ldots, j\}) & \leq(k+1) \cdot 2^{n-k-1} \sum_{j=k+1}^{n} m(\{1, \ldots, j\}) \\
& =(k+1) \cdot 2^{n-k-1}(1-\underline{P}(B)) .
\end{aligned}
$$


Now,

$$
\begin{aligned}
& \frac{k \cdot 2^{n-k} \underline{P}(B)}{k \cdot 2^{n-k} \underline{P}(B)+(k+1) \cdot 2^{n-k-1}(1-\underline{P}(B))} \geq \underline{P}(B) \\
& \Longleftrightarrow k \cdot 2^{n-k} \underline{P}(B) \geq\left(k \cdot 2^{n-k} \underline{P}(B)+(k+1) \cdot 2^{n-k-1}(1-\underline{P}(B))\right) \underline{P}(B) \\
& \Longleftrightarrow\left(k \cdot 2^{n-k}-(k+1) \cdot 2^{n-k-1}\right) \underline{P}(B) \geq\left(k \cdot 2^{n-k}-(k+1) \cdot 2^{n-k-1}\right) \underline{P}^{2}(B),
\end{aligned}
$$

and this last inequality holds because $f(j)=j \cdot 2^{n-j}$ is non-increasing and $\underline{P}(B) \in$ $[0,1]$.

Now, since we are assuming that the focal elements of $\underline{P}$ are among the sets $\{1, \ldots, k\}$ for $k=1, \ldots, n$, we deduce that for any other set $A$, if we let $k_{A}$ be the maximum $k \in\{1, \ldots, n\}$ such that $\{1, \ldots, k\} \subseteq A$,

$$
\underline{P}(A)=\underline{P}\left(\left\{1, \ldots, k_{A}\right\}\right) \leq \Psi(\underline{P})\left(\left\{1, \ldots, k_{A}\right\}\right) \leq \Psi(\underline{P})(A) .
$$

Thus, $\Psi(\underline{P})$ belongs to the core of $\underline{P}$.

As a summary, we conclude that for minitive functions the computation of both the Shapley and the normalized Banzhaf values can be simplified, and also that both of them belong to the core (Propositions 1 and 4).

\section{2-monotone lower probabilities}

From Proposition 1 we know that the Shapley value belongs to the core for 2monotone lower probabilities. In this section we investigate some particular cases of 2-monotone lower probabilities, such as coherent lower probabilities defined on spaces of cardinality 3 , probability intervals, distortion models or belief functions, to investigate if we can simplify the expression in Eq. (6). Also, we aim to see if in these particular cases the normalized Banzhaf value belongs to the core or not.

\subsection{2-monotone lower probabilities in a 3-element space}

Let us consider the particular case where the possibility space has three elements. It has been proven that in that case a lower probability is 2-monotone if and only if it is coherent (see ${ }^{27}$, Page 58). Moreover, in the case of cardinality three 2monotone lower probabilities are particular instances of probability intervals ${ }^{28}$, that is, they are uniquely determined by the constraints $[\underline{P}(\{i\}), \bar{P}(\{i\})]$. In other words, it suffices to know in this case the lower and upper limits on the gain of each player.

Our next proposition gives an alternative expression for the Shapley value in this case:

Proposition 5. Let $\underline{P}: \mathcal{P}(\{1,2,3\}) \rightarrow[0,1]$ be a 2-monotone lower probability. Then,

$$
\Phi(\underline{P})(i)=\frac{1}{3}+\frac{1}{2}(\underline{P}(\{i\})+\bar{P}(\{i\}))-\frac{1}{6} \sum_{l=1}^{3}(\underline{P}(\{l\})+\bar{P}(\{l\})) \quad \forall i \in \Omega .
$$


Proof. Given a permutation $\sigma$ of $\{1,2,3\}$, from Eq. (4) its associated extreme point $P_{\sigma}$ is given by:

$$
\begin{aligned}
& P_{\sigma}(\{\sigma(1)\})=\underline{P}(\{\sigma(1)\}), \\
& P_{\sigma}(\{\sigma(1), \sigma(2)\})=\underline{P}(\{\sigma(1), \sigma(2)\}), \\
& P_{\sigma}(\{\sigma(1), \sigma(2), \sigma(3)\})=\underline{P}(\{\sigma(1), \sigma(2), \sigma(3)\})=1 .
\end{aligned}
$$

Hence:

$$
\begin{aligned}
P_{\sigma}(\{\sigma(1)\}) & =\underline{P}(\{\sigma(1)\}), \\
P_{\sigma}(\{\sigma(2)\}) & =P_{\sigma}(\{\sigma(1), \sigma(2)\})-P_{\sigma}(\{\sigma(1)\}) \\
& =\underline{P}(\{\sigma(1), \sigma(2)\})-\underline{P}(\{\sigma(1)\})=1-\bar{P}(\{\sigma(3)\})-\underline{P}(\{\sigma(1)\}), \\
P_{\sigma}(\{\sigma(3)\}) & =P_{\sigma}(\{\sigma(1), \sigma(2), \sigma(3)\})-P_{\sigma}(\{\sigma(1), \sigma(2)\}) \\
& =1-\underline{P}(\{\sigma(1), \sigma(2)\})=\bar{P}(\{\sigma(3)\}),
\end{aligned}
$$

using twice the equality $\underline{P}(\{\sigma(1), \sigma(2)\})=1-\bar{P}\left(\{\sigma(1), \sigma(2)\}^{c}\right)=1-\bar{P}(\{\sigma(3)\})$. Thus, there are the following possibilities:

- If $\sigma(\{1\})=i$, then $P_{\sigma}(\{i\})=\underline{P}(\{i\})$;

- If $\sigma(\{2\})=i$, then $P_{\sigma}(\{i\})=1-\bar{P}(\{\sigma(3)\})-\underline{P}(\{\sigma(1)\})$;

- If $\sigma(\{3\})=i$, then $P_{\sigma}(\{i\})=\bar{P}(\{i\})$.

Therefore, since any of the previous cases appears for two permutations:

$$
\begin{aligned}
\Phi(\underline{P})(i) & =\frac{1}{n !} \sum_{\sigma} P_{\sigma}(\{i\}) \\
& =\frac{1}{6}(2 \underline{P}(\{i\})+2+2 \bar{P}(\{i\})-\underline{P}(\{j\})-\underline{P}(\{k\})-\bar{P}(\{j\})-\bar{P}(\{k\})),
\end{aligned}
$$

which can be equivalently be rewritten as

$$
\frac{1}{3}+\frac{1}{2}(\underline{P}(\{i\})+\bar{P}(\{i\}))-\frac{1}{6} \sum_{l=1}^{3}(\underline{P}(\{l\})+\bar{P}(\{l\})) .
$$

It is also possible to derive the above result by means of Eq. (6). Next, we provide a simple expression for the normalized Banzhaf value in the case of cardinality three.

Proposition 6. Let $\underline{P}: \mathcal{P}(\{1,2,3\}) \rightarrow[0,1]$ be a 2-monotone lower probability. Then, its normalized Banzhaf value is given by:

$$
\Psi(\underline{P})(i)=\frac{4 m(\{i\})+2 \sum_{j \neq i} m(\{i, j\})+m(\Omega)}{4-m(\Omega)} \quad \forall i \in \Omega .
$$

Proof. It follows from Eq. (7) that

$B(\underline{P})(1)=\frac{1}{4}(\underline{P}(\{1\})+\underline{P}(\{1,2\})-\underline{P}(\{2\})+\underline{P}(\{1,3\})-\underline{P}(\{3\})+1-\underline{P}(\{2,3\}))$, 
which in terms of the Möbius inverse is equal to

$$
\frac{4 m(\{1\})+2 m(\{1,2\})+2 m(\{1,3\})+m(\Omega)}{4} .
$$

Thus,

$$
\begin{aligned}
& B(\underline{P})(1)+B(\underline{P})(2)+B(\underline{P})(3) \\
& =\frac{4(m(\{1\})+m(\{2\})+m(\{3\})+m(\{1,2\})+m(\{1,3\})+m(\{2,3\}))+3 m(\Omega)}{4} \\
& =\frac{4-m(\Omega)}{4}=1-\frac{m(\Omega)}{4},
\end{aligned}
$$

from which the expression of the normalized Banzhaf value follows.

From the expression above we can derive that the normalized Banzhaf value always belongs to the core in the case of games with three players:

Proposition 7. Let $\underline{P}$ be a 2-monotone lower probability on $\Omega=\{1,2,3\}$ and let $\Psi(\underline{P})$ be its normalized Banzhaf value. Then $\Psi(\underline{P})$ belongs to $\mathcal{M}(\underline{P})$.

Proof. We need to establish that $\Psi(\underline{P})(i) \in[\underline{P}(\{i\}), \bar{P}(\{i\})]$ for every $i \in \Omega$. Let us prove it for $i=1$.

Let us first see that $\Psi(\underline{P})(1) \geq \underline{P}(\{1\})=m(\{1\})$. From Proposition 6,

$$
\Psi(\underline{P})(1)=\frac{4 m(\{1\})+2 m(\{1,2\})+2 m(\{1,3\})+m(\{1,2,3\})}{4-m(\{1,2,3\})} .
$$

Then, $\Psi(\underline{P})(1) \geq \underline{P}(\{1\})=m(\{1\})$ if and only if

$4 m(\{1\})+2 m(\{1,2\})+2 m(\{1,3\})+m(\{1,2,3\}) \geq 4 m(\{1\})-m(\{1,2,3\}) m(\{1\})$

or, equivalently, if and only if

$$
2 m(\{1,2\})+2 m(\{1,3\})+m(\{1,2,3\})(1+m(\{1\})) \geq 0 .
$$

2-monotonicity implies that $\underline{P}(\{1,2\}) \geq \underline{P}(\{1\})+\underline{P}(\{2\})$ and $\underline{P}(\{1,3\}) \geq \underline{P}(\{1\})+$ $\underline{P}(\{3\})$. Applying Eq. (1), we deduce that $m(\{1,2\}), m(\{1,3\}) \geq 0$. Now, we have two cases:

(1) If also $m(\{1,2,3\}) \geq 0$, then Eq. (11) trivially holds because all the terms are non-negative.

(2) Otherwise, from Theorem 2.7.4 in ${ }^{5}$, the inequality $\underline{P}(\{1,2\})+\underline{P}(\{2,3\}) \leq$ $1+\underline{P}(\{2\})$ holds, and it can be equivalently expressed in terms of the Möbius inverse as $0 \leq m(\{1,3\})+m(\{1,2,3\})$, again using Eq. (1). Since moreover $1+m(\{1\}) \leq 2$, we conclude that:

$$
\begin{array}{rl}
2 m(\{1,2\})+2 & m(\{1,3\})+m(\{1,2,3\})(1+m(\{1\})) \\
\geq & 2 m(\{1,2\})+2 m(\{1,3\})+2 m(\{1,2,3\}) \\
= & 2 m(\{1,2\})+2(m(\{1,3\})+m(\{1,2,3\})) \geq 0,
\end{array}
$$


because we have seen that both $m(\{1,2\})$ and $m(\{1,3\})+m(\{1,2,3\})$ are nonnegative.

We conclude that in both cases Eq. (11) holds, whence $\Psi(\underline{P})(1) \geq \underline{P}(\{1\})$.

Next, let us see that $\Psi(\underline{P})(1) \leq \bar{P}(\{1\})$. From Proposition 6, this inequality is equivalent to:

$$
\Psi(\underline{P})(1)=\frac{4 m(\{1\})+2 m(\{1,2\})+2 m(\{1,3\})+m(\{1,2,3\})}{4-m(\{1,2,3\})} \leq \bar{P}(\{1\}),
$$

which can also be expressed as:

$$
\begin{aligned}
4 m(\{1\}) & +2 m(\{1,2\})+2 m(\{1,3\})+m(\{1,2,3\}) \leq 4 \bar{P}(\{1\})-m(\{1,2,3\}) \bar{P}(\{1\}) \\
& =4(m(\{1\})+m(\{1,2\})+m(\{1,3\})+m(\{1,2,3\}))-m(\{1,2,3\}) \bar{P}(\{1\}),
\end{aligned}
$$

where in the last equality we have just expressed $\bar{P}(\{1\})$ in terms of $m$. The above inequality is equivalent to:

$$
2 m(\{1,2\})+2 m(\{1,3\})+m(\{1,2,3\})(3-\bar{P}(\{1\})) \geq 0 .
$$

Remember that, as we have already mentioned, $m(\{1,2\}), m(\{1,3\}) \geq 0$. We have two cases:

(1) If $m(\{1,2,3\}) \geq 0$, the above equation holds trivially because all the terms are non-negative.

(2) Otherwise, use that

$$
-m(\{1,2,3\})(3-\bar{P}(\{1\})) \leq-3 m(\{1,2,3\}) \leq 2 m(\{1,2\})+2 m(\{1,3\}),
$$

since, with an analogous reasoning to that above, $m(\{1,2\})+m(\{1,2,3\}) \geq 0$ and $m(\{1,3\})+m(\{1,2,3\}) \geq 0$.

In both cases, we conclude that Eq. (12) holds, and therefore $\Psi(\underline{P})(1) \leq \bar{P}(\{1\})$.

As we shall see, this result does not extend to greater cardinalities (see Examples 1 or 3 later on). In other words, this is the largest cardinality of a possibility space where the Banzhaf value is always guaranteed to belong to the core for 2monotone lower probabilities.

\subsection{Probability intervals}

One of the simplest cases of 2-monotone lower probability is that of probability intervals ${ }^{28}$. A probability interval is given by $\mathcal{I}=\left\{\left[l_{i}, u_{i}\right]: i=1, \ldots, n\right\}$, where $0 \leq l_{i} \leq u_{i} \leq 1$ and $\left[l_{i}, u_{i}\right]$ represents that the unknown probability of $i$ lies between $l_{i}$ and $u_{i}$. Any probability interval defines a core by:

$\mathcal{M}(\mathcal{I})=\left\{P: \mathcal{P}(\Omega) \rightarrow[0,1]\right.$ probability measure $\left.: l_{i} \leq P(\{i\}) \leq u_{i} \quad \forall i=1, \ldots, n\right\}$. 
Using the probabilistic information of the core, we can define a lower and an upper probability by:

$$
\underline{P}(A)=\inf _{P \in \mathcal{M}(\mathcal{I})} P(A), \quad \bar{P}(A)=\sup _{P \in \mathcal{M}(\mathcal{I})} P(A) \quad \forall A \subseteq \Omega .
$$

From the point of view of coalitional game theory, a model of probability intervals means that we are only given the information about the coalitions formed by one, or all but one player, and that the information about the other coalitions is the one we can derive from these.

In ${ }^{28}$, it is proven that $\underline{P}, \bar{P}$ are coherent if and only if $\underline{P}(\{i\})=l_{i}$ and $\bar{P}(\{i\})=$ $u_{i}$ for any $i=1, \ldots, n$. In such a case, $\underline{P}$ and $\bar{P}$ can be computed, for any $A$, by:

$$
\underline{P}(A)=\max \left\{\sum_{i \in A} l_{i}, 1-\sum_{i \notin A} u_{i}\right\}, \quad \bar{P}(A)=\min \left\{\sum_{i \in A} u_{i}, 1-\sum_{i \notin A} l_{i}\right\} .
$$

On the one hand, when the lower probability induced by a probability interval by means of Eq. (13) is coherent, it is also 2-monotone. Therefore, from Proposition 1 the Shapley value can be computed using Eq. (6) and it belongs to the core.

We may think that the normalized Banzhaf value also belongs to the core. However, this is not true in general, as our next example shows.

Example 1. Consider the probability interval $\mathcal{I}$ in $\Omega=\{1,2,3,4\}$ given by:

$$
l_{1} \in[0.2,0.2], \quad l_{2} \in[0.1,0.4], \quad l_{3} \in[0.2,0.3], \quad l_{4} \in[0.1,0.4] .
$$

The Banzhaf value is given by: $B(\underline{P})=\left(\frac{1.6}{8}, \frac{2.2}{8}, \frac{2.2}{8}, \frac{2.2}{8}\right)$, and therefore the normalized Banzhaf value is given by: $\Psi(\underline{P})=\left(\frac{1.6}{8.2}, \frac{2.2}{8.2}, \frac{2.2}{8.2}, \frac{2.2}{8.2}\right)$. Whence, $\Psi(\underline{P})(1)<$ $l_{1}=u_{1}$, and therefore $\Psi(\underline{P}) \notin \mathcal{M}(\mathcal{I})$. This example also shows that the condition $\sum_{i=1}^{n} B(\underline{P})(i) \leq 1$ is not satisfied.

On the other hand, in our previous section we considered the case of threeelement spaces, and showed that in that particular case any coherent lower probability is also a probability interval. Moreover, Proposition 7 allows us to conclude that for any probability interval in a 3-element space both the Shapley and normalized Banzhaf values belong to the core. Our example above shows that the normalized Banzhaf value need not belong to the core for spaces of higher cardinality.

\subsection{Lower probabilities induced by a distortion model}

Two particular cases of 2-monotone lower probabilities are those induced by a Parimutuel model (PMM for short) or an $\varepsilon$-contamination model; these two cases are usually referred to as instances of distortion models. The PMM has its origin in horse racing. It considers a probability $P_{0}$ on $\mathcal{P}(\Omega)$ and a distortion value $\delta>0$. Using $P_{0}$ and $\delta$, the PMM defines a lower probability $\underline{P}$ by ${ }^{5,29,30,31}$ :

$$
\underline{P}(A)=\max \left\{(1+\delta) P_{0}(A)-\delta, 0\right\} .
$$


From ${ }^{29}$, the lower probability $\underline{P}$ induced by a PMM is in particular a probability interval, and as a consequence also 2-monotone. Thus, Proposition 1 implies that the Shapley value coincides with the center of gravity of the core.

The same happens with $\varepsilon$-contamination models. There we consider a probability $P_{0}$ and a contamination value called $\varepsilon \in(0,1)$, that represents the distortion made to $P_{0}$. The $\varepsilon$-contamination model defines a lower probability by:

$$
\underline{P}(A)=(1-\varepsilon) P_{0}(A)+\varepsilon \underline{P}_{\Omega}(A),
$$

where $\underline{P}_{\Omega}$ is the vacuous lower probability ${ }^{\mathrm{c}}$ that assigns the value 1 to $\Omega$ and 0 otherwise. In other words, $\underline{P}(A)=(1-\varepsilon) P_{0}(A)$ for every $A \subsetneq \Omega$ and $\underline{P}(\Omega)=1$. The lower probability associated with an $\varepsilon$-contamination model is known to be, not only 2-monotone, but also completely monotone, that is, $\underline{P}$ is a belief function. This follows for instance from Theorems 5 and 11 of ${ }^{32}$, taking into account that complete monotonicity is preserved by convex combinations. $\epsilon$-contamination models are sometimes referred to as linear-vacuous mixtures (see Section 2.9 .2 of ${ }^{5}$ ), and can be used for instance in a cautious Bayesian approach where the expert has incomplete confidence in the assessment $P_{0}$.

A possible interpretation of distortion models from the point of view of coalitional game theory would correspond to the case where we have precise information about the reward associated with each player, in terms of a probability measure $P_{0}$, but, due to a number of factors, there is some probability of error in its estimation (measured by the coefficients $\delta$ and $\varepsilon$ ), that transforms $P_{0}$ into a set of possible probability measures, that we summarize by means of its lower envelope $\underline{P}$.

Although one may think that for a distortion model based on the probability $P_{0}$, both the Shapley and normalized Banzhaf values coincide with $P_{0}$, our next example shows that this is not the case:

Example 2. Consider the probability $P_{0}$ on $\{1,2,3\}$ given by $P_{0}(\{1\})=0.1$, $P_{0}(\{2\})=0.2$ and $P_{0}(\{3\})=0.7$. Take $\delta=\varepsilon=0.3$, and denote by $\underline{P}_{\delta}$ and $\underline{P}_{\varepsilon}$ the lower probabilities induced by the PMM and $\varepsilon$-contamination, respectively. Using Eqs. (14) and (15), $\underline{P}_{\delta}$ and $\underline{P}_{\varepsilon}$ are given by:

\begin{tabular}{c|c|c|c|c|c|c|c}
$A$ & $\{1\}$ & $\{2\}$ & $\{3\}$ & $\{1,2\}$ & $\{1,3\}$ & $\{2,3\}$ & $\{1,2,3\}$ \\
\hline$\underline{P}_{\delta}(A)$ & 0 & 0 & 0.61 & 0.09 & 0.74 & 0.87 & 1 \\
$\underline{P}_{\varepsilon}(A)$ & 0.07 & 0.14 & 0.49 & 0.21 & 0.56 & 0.63 & 1
\end{tabular}

We deduce from Eq. (4) that the extreme points of $\mathcal{M}\left(\underline{P}_{\delta}\right)$ and $\mathcal{M}\left(\underline{P}_{\varepsilon}\right)$ are given

${ }^{\mathrm{c}}$ The vacuous lower probability is an instance of a unanimity game (see Section 2.8.2 of ${ }^{1}$ ), also referred to as simple support functions by Shafer ${ }^{3}$. 
by:

\begin{tabular}{c|cc}
$\sigma$ & $P_{\sigma}$ for $\mathcal{M}\left(\underline{P}_{\delta}\right)$ & $P_{\sigma}$ for $\mathcal{M}\left(\underline{P}_{\varepsilon}\right)$ \\
\hline$(1,2,3)$ & $(0,0.09,0.91)$ & $(0.07,0.14,0.79)$ \\
$(1,3,2)$ & $(0,0.26,0.74)$ & $(0.07,0.44,0.49)$ \\
$(2,1,3)$ & $(0.09,0,0.91)$ & $(0.07,0.14,0.79)$ \\
$(2,3,1)$ & $(0.13,0,0.87)$ & $(0.37,0.14,0.49)$ \\
$(3,1,2)$ & $(0.13,0.26,0.61)$ & $(0.07,0.44,0.49)$ \\
$(3,2,1)$ & $(0.13,0.26,0.61)$ & $(0.37,0.14,0.49)$
\end{tabular}

The centers of gravity of $\mathcal{M}\left(\underline{P}_{\delta}\right)$ and $\mathcal{M}\left(\underline{P}_{\varepsilon}\right)$ are $\Phi\left(\underline{P}_{\delta}\right)=(0.08,0.145,0.775)$ and $\Phi\left(\underline{P}_{\epsilon}\right)=(0.17,0.24,0.59)$, respectively, and none of them coincide with $P_{0}$.

Similarly, the normalized Banzhaf values are given by $\Psi\left(\underline{P}_{\delta}\right)=\left(\frac{0.35}{4.09}, \frac{0.61}{4.09}, \frac{3.13}{4.09}\right)$ and $\Psi\left(\underline{P}_{\delta}\right)=\left(\frac{0.58}{3.7}, \frac{0.86}{3.7}, \frac{2.26}{3.7}\right)$, which do not coincide with $P_{0}$ either.

We now consider the PMM in the particular case $P_{0}(\{i\})<1$ and $\delta<\frac{P_{0}(\{i\})}{1-P_{0}(\{i\})}$ for any $i=1, \ldots, n$. As we shall see, this corresponds to the case where $\underline{P}$ is strictly positive for any non-empty set. In that case we can give a simple expression for the Shapley and the normalized Banzhaf values.

Proposition 8. Let $\underline{P}$ be the lower probability associated with a PMM induced by $P_{0}, \delta$, and assume that $\delta<\frac{P_{0}(\{i\})}{1-P_{0}(\{i\})}$ for any $i=1, \ldots, n$. Then the Shapley value is given by:

$$
\Phi(\underline{P})(i)=(1+\delta) P_{0}(\{i\})-\frac{\delta}{n},
$$

while the normalized Banzhaf value is

$$
\Psi(\underline{P})(i)=\frac{(1+\delta) P_{0}(\{i\})-\frac{\delta}{2^{n-1}}}{k}, \text { where } k=(1+\delta)-\frac{n \delta}{2^{n-1}} .
$$

Moreover, both the Shapley and normalized Banzhaf values belong to the core.

Proof. First of all, since $\delta<\frac{P_{0}(\{i\})}{1-P_{0}(\{i\})}$ for any $i=1, \ldots, n$, it holds that:

$(1+\delta) P_{0}(\{i\})-\delta=\delta\left(P_{0}(\{i\})-1\right)+P_{0}(\{i\})>\frac{P_{0}(\{i\})}{1-P_{0}(\{i\})}\left(P_{0}(\{i\})-1\right)+P_{0}(\{i\})=0$.

Thus, $\underline{P}(\{i\})>0$ for any $i=1, \ldots, n$, and as a consequence for any $A \neq \emptyset$, the monotonicity of $\underline{P}$ (see Section 2.7.4 in ${ }^{5}$ ) implies that $\underline{P}(A)>0$. By conjugacy, we obtain $\bar{P}(A)<1$ for every $A \neq \Omega$.

To obtain the expression for the Shapley value, we use Eq. (5). For any permutation $\sigma$ and any $j<n$, from Eq. (4) it holds that:

$P_{\sigma}(\{\sigma(1), \ldots, \sigma(j)\})=\underline{P}(\{\sigma(1), \ldots, \sigma(j)\})=(1+\delta) P_{0}(\{\sigma(1), \ldots, \sigma(j)\})-\delta>0$.

From this we deduce that

$$
P_{\sigma}(\{\sigma(1)\})=\underline{P}(\{\sigma(1)\}) \text { and } P_{\sigma}(\{\sigma(i)\})=(1+\delta) P_{0}(\{\sigma(i)\}) \quad \forall i=2, \ldots, n .
$$


In other words,

$$
P_{\sigma}(\{i\})= \begin{cases}\bar{P}(\{i\})=(1+\delta) P_{0}(\{i\}) & \text { if } i \neq \sigma(1) . \\ \underline{P}(\{i\})=(1+\delta) P_{0}(\{i\})-\delta & \text { if } i=\sigma(1) .\end{cases}
$$

Note also that $i=\sigma(1)$ for $(n-1)$ ! permutations, while $i \neq \sigma(1)$ for $n !-(n-1) !=$ $(n-1) !(n-1)$ permutations. Therefore:

$$
\begin{aligned}
\Phi(\underline{P})(i) & =\frac{1}{n !} \sum_{\sigma \in S^{\Omega}} P_{\sigma}(\{i\}) \\
& =\frac{1}{n !}\left((n-1) !\left((1+\delta) P_{0}(\{i\})-\delta\right)+(n-1) !(n-1)(1+\delta) P_{0}(\{i\})\right) \\
& =\frac{1}{n}\left((1+\delta) P_{0}(\{i\})-\delta+(n-1)(1+\delta) P_{0}(\{i\})\right)=(1+\delta) P_{0}(\{i\})-\frac{\delta}{n} .
\end{aligned}
$$

On the other hand, if we take into account that $\underline{P}(A)=(1+\delta) P_{0}(A)-\delta$ for every non-empty $A$, we obtain that the Banzhaf value on $i$ is given by

$$
\begin{aligned}
B(\underline{P})(i) & =\frac{1}{2^{n-1}} \sum_{i \notin A}(\underline{P}(A \cup\{i\})-\underline{P}(A)) \\
= & \frac{P}{2^{n-1}}+\frac{1}{2^{n-1}} \sum_{i \notin A \neq \emptyset}(\underline{P}(A \cup\{i\})-\underline{P}(A)) \\
= & \frac{(1+\delta) P_{0}(\{i\})-\delta}{2^{n-1}}+\frac{2^{n-1}-1}{2^{n-1}}(1+\delta) P_{0}(\{i\})=(1+\delta) P_{0}(\{i\})-\frac{\delta}{2^{n-1}} .
\end{aligned}
$$

This implies that $\sum_{i \in \Omega} B(\underline{P})(i)=(1+\delta)-\frac{n \delta}{2^{n-1}}$, from which the expression of the normalized Banzhaf value follows.

It only remains to see that both the Shapley and normalized Banzhaf values belong to $\mathcal{M}(\underline{P})$. On the one hand, since the lower probability induced by the PMM is 2-monotone, it follows from Proposition 1 that the Shapley value belongs to the core. Let us see that this is also the case for the normalized Banzhaf value.

For this aim, we shall prove that $\Psi(\underline{P})(A) \leq \bar{P}(A)$ for every $A \subseteq \Omega$, where $\bar{P}$ denotes the upper probability of the PMM, given by:

$$
\bar{P}(A)=\min \left\{(1+\delta) P_{0}(A), 1\right\} .
$$

First of all, the condition $\delta<\frac{P_{0}(\{i\})}{1-P_{0}(\{i\})}$ ensures that (see Lemma 1 in ${ }^{29}$ ):

$$
\bar{P}(A)=(1+\delta) P_{0}(A)=\sum_{i \in A} \bar{P}(\{i\}) \forall A \subsetneq \Omega .
$$

We begin by proving that $\Psi(\underline{P})(i) \leq \bar{P}(\{i\})$ for any $i=1, \ldots, n$. This holds if and only if:

$$
\frac{(1+\delta) P_{0}(\{i\})-\frac{\delta}{2^{n-1}}}{(1+\delta)-\frac{n \delta}{2^{n-1}}} \leq(1+\delta) P_{0}(\{i\})
$$


which is equivalent to:

$$
P_{0}(\{i\})\left((1+\delta)-(1+\delta)^{2}+\frac{n \delta}{2^{n-1}}(1+\delta)\right) \leq \frac{\delta}{2^{n-1}},
$$

and therefore to:

$$
(1+\delta) P_{0}(\{i\})\left(\frac{n}{2^{n-1}}-1\right) \leq \frac{1}{2^{n-1}},
$$

and this inequality holds because for $n \geq 1, \frac{n}{2^{n-1}}-1 \leq 0$. Thus, $\Psi(\underline{P})(i) \leq \bar{P}(\{i\})$. Now, given $A \subsetneq \Omega$, it holds that

$$
\Psi(\underline{P})(A)=\sum_{i \in A} \Psi(\underline{P})(i) \leq \sum_{i \in A} \bar{P}(\{i\})=\bar{P}(A) .
$$

Since trivially $\Psi(\underline{P})(\Omega)=\bar{P}(\Omega)=1$, we conclude that $\Psi(\underline{P}) \leq \bar{P}$ and therefore $\Psi(\underline{P})$ belongs to the core.

Next result shows that for $\varepsilon$-contamination models we can also obtain the Shapley and Banzhaf values, from expressions similar to those of Eqs. (16) and (17).

Proposition 9. Let $\underline{P}$ be the lower probability associated with an $\varepsilon$-contamination induced by $P_{0}, \varepsilon$. Then the Shapley value is given by:

$$
\Phi(\underline{P})(i)=(1-\varepsilon) P_{0}(\{i\})+\frac{\varepsilon}{n},
$$

while the normalized Banzhaf value is

$$
\Psi(\underline{P})(i)=\frac{(1-\varepsilon) P_{0}(\{i\})+\frac{\epsilon}{2^{n-1}}}{k}, \text { where } k=(1-\varepsilon)+\frac{n \varepsilon}{2^{n-1}} .
$$

Moreover, both the Shapley and normalized Banzhaf values belong to the core.

Proof. To obtain the expression for the Shapley value, we use again that, since the $\varepsilon$-contamination model is completely monotone, we can apply Eq. (5). First of all, note that for $A \neq \Omega$, it holds that:

$$
\underline{P}(A)=(1-\varepsilon) P_{0}(A)=(1-\varepsilon) \sum_{i \in A} P_{0}(\{i\}) .
$$

This implies that for any permutation $\sigma$ and $j<n$, from Eq. (4) it holds that:

$$
P_{\sigma}(\{\sigma(1), \ldots, \sigma(j)\})=(1-\varepsilon) P_{0}(\{\sigma(1), \ldots, \sigma(j)\}) .
$$

From this we deduce that for $j<n$ :

$$
\begin{aligned}
P_{\sigma}(\{\sigma(j)\}) & =P_{\sigma}(\{\sigma(1), \ldots, \sigma(j)\})-P_{\sigma}(\{\sigma(1), \ldots, \sigma(j-1)\}) \\
& =(1-\varepsilon) P_{0}(\{\sigma(1), \ldots, \sigma(j)\})-(1-\varepsilon) P_{0}(\{\sigma(1), \ldots, \sigma(j-1)\}) \\
& =(1-\varepsilon) P_{0}(\{\sigma(j)\}),
\end{aligned}
$$


and also:

$$
\begin{aligned}
P_{\sigma}(\{\sigma(n)\}) & =P_{\sigma}(\{\sigma(1), \ldots, \sigma(n)\})-P_{\sigma}(\{\sigma(1), \ldots, \sigma(n-1)\}) \\
& =(1-\varepsilon) P_{0}(\{\sigma(1), \ldots, \sigma(n)\})+\varepsilon-(1-\varepsilon) P_{0}(\{\sigma(1), \ldots, \sigma(n-1)\}) \\
& =(1-\varepsilon) P_{0}(\{\sigma(n)\})+\varepsilon .
\end{aligned}
$$

Then, we obtain that:

$$
P_{\sigma}(\{i\})= \begin{cases}(1-\varepsilon) P_{0}(\{i\}) & \text { if } i \neq \sigma(n) . \\ (1-\varepsilon) P_{0}(\{i\})+\varepsilon & \text { if } i=\sigma(n) .\end{cases}
$$

Also, $i=\sigma(n)$ for $(n-1)$ ! permutations, while $i \neq \sigma(n)$ for $n !-(n-1) !=$ $(n-1) !(n-1)$ permutations. Therefore:

$$
\begin{aligned}
\Phi(\underline{P})(i) & =\frac{1}{n !} \sum_{\sigma \in S^{\Omega}} P_{\sigma}(\{i\}) \\
& =\frac{1}{n !}\left((n-1) !\left((1-\varepsilon) P_{0}(\{i\})+\varepsilon\right)+(n-1) !(n-1)(1-\varepsilon) P_{0}(\{i\})\right) \\
& =\frac{1}{n}\left((1-\varepsilon) P_{0}(\{i\})+\varepsilon+(n-1)(1-\varepsilon) P_{0}(\{i\})\right)=(1-\varepsilon) P_{0}(\{i\})+\frac{\varepsilon}{n} .
\end{aligned}
$$

To compute the normalized Banzhaf value, note that if $A \cup\{i\} \neq \Omega$, it holds that:

$$
\begin{aligned}
& \underline{P}(A \cup\{i\})=(1-\varepsilon)\left(P_{0}(\{i\})+\sum_{j \in A} P_{0}(\{j\})\right) \\
& \underline{P}(A)=(1-\varepsilon) \sum_{j \in A} P_{0}(\{j\})
\end{aligned}
$$

and therefore $\underline{P}(A \cup\{i\})-\underline{P}(A)=(1-\varepsilon) P_{0}(\{i\})$. On the other hand, if $A \cup\{i\}=\Omega$, it holds that:

$$
\begin{aligned}
& \underline{P}(A \cup\{i\})=1 \\
& \underline{P}(A)=(1-\varepsilon) \sum_{j \in A} P_{0}(\{j\})=(1-\varepsilon)-P_{0}(\{i\})(1-\varepsilon)
\end{aligned}
$$

and therefore $\underline{P}(A \cup\{i\})-\underline{P}(A)=\varepsilon+P_{0}(\{i\})(1-\varepsilon)$.

Taking into account that there are $2^{n-1}-1$ sets $A$ such that $A \cup\{i\} \neq \Omega$, we can compute the Banzhaf value:

$$
\begin{aligned}
B(\underline{P})(i) & =\frac{1}{2^{n-1}} \sum_{A \nsupseteq\{i\}}(\underline{P}(A \cup\{i\})-\underline{P}(A)) \\
& =\frac{1}{2^{n-1}}\left(\left(2^{n-1}-1\right)(1-\varepsilon) P_{0}(\{i\})+\varepsilon+(1-\varepsilon) P_{0}(\{i\})\right) \\
& =(1-\varepsilon) P_{0}(\{i\})+\frac{\varepsilon}{2^{n-1}} .
\end{aligned}
$$

Finally, taking into account that

$$
\sum_{i=1}^{n} B(\underline{P})(i)=(1-\varepsilon)+\frac{n \varepsilon}{2^{n-1}},
$$

we can normalize $B(\underline{P})(i)$ and we obtain the value on Eq. (18). 
It only remains to see that both the Shapley and normalized Banzhaf values belong to the core. On the one hand, since the lower probability induced by the $\varepsilon$ contamination model is 2-monotone, it follows from Proposition 1 that the Shapley value belongs to the core. Let us see that the normalized Banzhaf value also belongs to $\mathcal{M}(\underline{P})$. For this aim, let us see that $\Psi(\underline{P})(i) \geq \underline{P}(\{i\})$ for any $i=1, \ldots, n$ :

$$
\begin{aligned}
\Psi(\underline{P})(i) \geq \underline{P}(\{i\}) & \Longleftrightarrow \frac{(1-\varepsilon) P_{0}(\{i\})+\frac{\varepsilon}{2^{n-1}}}{(1-\varepsilon)+\frac{n \varepsilon}{2^{n-1}}} \geq(1-\varepsilon) P_{0}(\{i\}) \\
& \Longleftrightarrow(1-\varepsilon) P_{0}(\{i\})\left[\varepsilon-\frac{n \varepsilon}{2^{n-1}}\right]+\frac{\varepsilon}{2^{n-1}} \geq 0,
\end{aligned}
$$

The last inequality holds because $\frac{n}{2^{n-1}}-1 \leq 0$ for $n \geq 1$, so $\varepsilon-\frac{n \varepsilon}{2^{n-1}} \geq 0$, and as a consequence $\Psi(\underline{P})(i) \geq \underline{P}(\{i\})$; if we now consider $A \subsetneq \Omega$, it holds that

$$
\Psi(\underline{P})(A)=\sum_{i \in A} \Psi(\underline{P})(i) \geq \sum_{i \in A} \underline{P}(\{i\})=\underline{P}(A),
$$

and since $\Psi(\underline{P})(\Omega)=\underline{P}(\Omega)=1$ we conclude that $\Psi(\underline{P})$ belongs to $\mathcal{M}(\underline{P})$.

A common choice for $P_{0}$ in a distortion model is the uniform distribution (see for example $\left.{ }^{33,34}\right)$. Next result shows that for the $\varepsilon$-contamination model and for the PMM with small enough values of $\delta$, the Shapley and normalized Banzhaf values coincide with $P_{0}$ if and only if $P_{0}$ follows a uniform distribution.

Corollary 2. Let $\underline{P}$ be the lower probability associated with either a PMM induced by $P_{0}, \delta$, where $\delta$ satisfies $\delta<\frac{P_{0}(\{i\})}{1-P_{0}(\{i\})}$ for any $i=1, \ldots, n$, or an $\varepsilon$-contamination model. Then the following are equivalent:

(1) $\Phi(\underline{P})=P_{0}$.

(2) $\Psi(\underline{P})=P_{0}$.

(3) $P_{0}(\{i\})=\frac{1}{n} \forall i \in \Omega$.

Proof. Assume that $\underline{P}$ is the PMM defined from $P_{0}, \delta$. Since $\delta$ satisfies the condition of Proposition 8, we deduce from Eq. (16) that:

$$
\Phi(\underline{P})(i)=(1+\delta) P_{0}(\{i\})-\frac{\delta}{n}=P_{0}(\{i\}) \Longleftrightarrow P_{0}(\{i\})=\frac{1}{n} .
$$

Similarly, by Eq. (17),

$$
\begin{aligned}
& \Psi(\underline{P})(i)=P_{0}(\{i\}) \\
& \Leftrightarrow(1+\delta) P_{0}(\{i\})-\frac{\delta}{2^{n-1}}=\left((1+\delta)-\frac{n \delta}{2^{n-1}}\right) P_{0}(\{i\}) \Leftrightarrow P_{0}(\{i\})=\frac{1}{n} .
\end{aligned}
$$

Next, if $\underline{P}$ is an $\varepsilon$-contamination model defined from $P_{0}, \varepsilon$, we deduce from Proposition 9 that

$$
\Phi(\underline{P})(i)=(1-\varepsilon) P_{0}(\{i\})+\frac{\varepsilon}{n}=P_{0}(\{i\}) \Longleftrightarrow P_{0}(\{i\})=\frac{1}{n} .
$$


With respect to the normalized Banzhaf value, applying Proposition 9,

$$
\Psi(\underline{P})(i)=P_{0}(\{i\}) \Longleftrightarrow(1-\varepsilon) P_{0}(\{i\})+\frac{\varepsilon}{2^{n-1}}=(1-\varepsilon) P_{0}(\{i\})+P_{0}(\{i\}) \frac{n \varepsilon}{2^{n-1}},
$$

which is also equivalent to $P_{0}(\{i\})=\frac{1}{n}$.

In fact, for the PMM we easily derive from the symmetry axiom satisfied by the Shapley and the Banzhaf values that, if $P_{0}$ is the uniform probability measure, then it coincides with the Shapley value of the PMM $\left(P_{0}, \delta\right)$ irrespective of the value of $\delta$; to see that the converse is not true in general, i.e., that $\Phi$ can be the uniform probability measure for other PMM $\left(P_{0}, \delta\right)$, it suffices to consider that $\mathcal{M}(\underline{P})$ is the set of all probability measures for $\underline{P}=\left(P_{0}, \delta\right)$ provided $\delta$ is large enough (specifically, when $\delta \geq \frac{1}{P_{0}\left(A^{c}\right)}$ for every $A \neq \Omega$ ): in that case $\Phi$ becomes the uniform distribution. Similar comments apply to the normalized Banzhaf value.

The key here is that, even if we may think that the distortion made with the PMM and the $\epsilon$-contamination models is uniform, it has implications in the probability transformations, that do not return the probability measure we started with, except when this measure is uniform itself.

\subsection{Belief functions}

In the particular case when $\underline{P}$ is completely monotone (that is, a belief function), we also have that the Shapley value of $\underline{P}$ belongs to the core $\mathcal{M}(\underline{P})$. Interestingly, the same property does not hold for the normalized Banzhaf value, as the following example shows:

Example 3. Let $\Omega=\{1,2,3,4\}$, and consider the belief function associated with the basic probability assignment given by $m(\{1\})=m(\{2,3,4\})=0.5$, and $m(A)=$ 0 for any other $A$. Then it follows from Eq. (7) that

$$
B(\underline{P})(1)=0.5, B(\underline{P})(2)=B(\underline{P})(3)=B(\underline{P})(4)=\frac{1}{8} .
$$

As a consequence, the probability mass function of the normalized Banzhaf value is given by $\Psi(\underline{P})=\left(\frac{4}{7}, \frac{1}{7}, \frac{1}{7}, \frac{1}{7}\right)$. However, this does not belong to the core of $\underline{P}$ : we have that $\Psi(\underline{P})(\{2,3,4\})=\frac{3}{7}<0.5=\underline{P}(\{2,3,4\})$.

For comparison, in this case Eq. (3) tells us that $\Phi(\underline{P})=\left(\frac{3}{6}, \frac{1}{6}, \frac{1}{6}, \frac{1}{6}\right)$.

This means that the result we have established in Proposition 4 does not extend to arbitrary belief functions. It also illustrates the difference between the Shapley and the normalized Banzhaf values.

One property of the Banzhaf value when $\underline{P}$ is a belief function is that the sum of the payoffs of the individual players never exceeds the total payoff, as the following proposition shows:

Proposition 10. Let $\underline{P}$ be a belief function, and let $B(\underline{P})$ be its associated Banzhaf value. Then $\sum_{i=1}^{n} B(\underline{P})(i) \leq 1$. 


\section{Proof.}

From Eq. (7), it follows that, for every $i \in \Omega$,

$$
\begin{aligned}
B(\underline{P})(i)=\frac{1}{2^{n-1}} \sum_{A \nsupseteq\{i\}}(\underline{P}(A \cup\{i\})-\underline{P}(A)) & =\frac{1}{2^{n-1}} \sum_{A \nsupseteq\{i\}} \sum_{B \subseteq A} m(B \cup\{i\}) \\
& =\frac{1}{2^{n-1}} \sum_{B \nsupseteq\{i\}} m(B \cup\{i\}) 2^{n-1-|B|},
\end{aligned}
$$

where last equality holds because any set $B$ that does not include $i$ can be included in $2^{n-1-|B|}$ subsets $A$ of $\Omega \backslash\{i\}$. Then:

$$
\sum_{i \in \Omega} B(\underline{P})(i)=\frac{1}{2^{n-1}} \sum_{B \nsupseteq\{i\}} m(B \cup\{i\}) 2^{n-1-|B|}=\frac{1}{2^{n-1}} \sum_{B \neq \Omega} \sum_{i \notin B} m(B \cup\{i\}) 2^{n-1-|B|} .
$$

Now, if we take into account that there is a one-to-one correspondence between the subsets $B$ of $\Omega \backslash\{i\}$ and those subsets $C$ of $\Omega$ that include $i$, Eq. (19) can be equivalently expressed by:

$$
\sum_{i \in \Omega} B(\underline{P})(i)=\frac{1}{2^{n-1}} \sum_{C \neq \emptyset} \sum_{j \in C} m(C) 2^{n-|C|}=\frac{1}{2^{n-1}} \sum_{C \neq \emptyset} m(C) 2^{n-|C|}|C|,
$$

where the last equality follows because fixed a set $C \neq \emptyset, m(C) 2^{n-|C|}$ does not depend on the chosen element $j \in C$.

Now, since $k \leq 2^{k-1}$ for every $k \geq 1$ and since any belief function $\underline{P}$ satisfies that $m(C) \geq 0$ for any subset $C$, we deduce that

$$
\frac{1}{2^{n-1}} \sum_{C \neq \emptyset} m(C) 2^{n-|C|}|C| \leq \sum_{C \neq \emptyset} m(C) \leq 1 .
$$

To see that this does not hold for 2-monotone lower probabilities that are not completely monotone, note that in Example 2 it holds that $\sum_{i=1}^{3} B\left(\underline{P}_{\delta}\right)(i)=\frac{4.09}{4}>$ 1. This can also be seen using Proposition 6 .

One instance of belief functions correspond to unanimity games, which are those for which there is some set $S \subseteq \Omega$ such that

$$
\underline{P}(A)=\left\{\begin{array}{l}
1 \text { if } S \subseteq A \\
0 \text { otherwise. }
\end{array}\right.
$$

This follows because $\underline{P}$ is a belief function with only one focal set: $m(S)=1$ and $m(A)=0$ for any $A \neq S$. The expressions of the Shapley and normalized Banzhaf values are given in the following proposition:

Proposition 11. Let $\underline{P}$ be the unanimity game associated with $S$. Then

$$
\Psi(\underline{P})(i)=\Phi(\underline{P})(i)= \begin{cases}\frac{1}{|S|} & \text { if } i \in S \\ 0 & \text { otherwise. }\end{cases}
$$


Proof. The formula for the Shapley value can be found in Lemma XII.1.5 of ${ }^{35}$. With respect to the normalized Banzhaf value, it follows from Eq. (7) that

$$
B(\underline{P})(i)=\left\{\begin{array}{l}
\frac{1}{2^{|S|}} \text { if } i \in S \\
0 \text { otherwise }
\end{array}\right.
$$

from which the expression in the statement follows.

The same expressions can also be obtained taking into account that the unanimity game is also minitive, and therefore we can use the results from Section 3. Moreover, it follows from ${ }^{5}$ that any 0-1 valued lower probability (called simple game in cooperative game theory) is coherent if and only if there is a filter ${ }^{\mathrm{d}}$ of subsets $\mathcal{F}$ of $\Omega$ such that $\underline{P}(A)=1$ if $A \in \mathcal{F}$, and $\underline{P}(A)=0$ otherwise. When $\Omega$ is finite, as is the case in this paper, any filter of subsets of $\Omega$ is fixed, which means that any coherent simple game corresponds to a unanimity game, and therefore that we can use Proposition 11 to compute the Shapley and normalized Banzhaf values. We shall show in Remark 2 that the result does not extend to simple games that are not coherent.

\section{Coherent lower probabilities}

We consider next the case of coherent lower probabilities. These correspond to exact games within cooperative game theory. It was established by Baroni and Vicig (see ${ }^{6}$, Proposition 5) in terms of the pignistic transformation, that the Shapley value of a coherent lower probability need not be an element of the core or, in other words, that Proposition 1 does not extend to coherent lower probabilities. The very same example allows us to show that the normalized Banzhaf value need not belong to the core, either:

Example 4. Consider $\Omega=\{1,2,3,4,5\}$, and let $\underline{P}$ be the coherent lower probability that is the lower envelope of the probability measures with mass functions

$$
\begin{aligned}
& P_{1}=(0.49,0.35,0.12,0.01,0.03) \\
& P_{2}=(0.14,0.03,0.07,0.36,0.40) \\
& P_{3}=(0.36,0.05,0.29,0.14,0.16)
\end{aligned}
$$

Baroni and Vicig (see Proposition 5 in ${ }^{6}$ ) showed that the Shapley value of $\underline{P}$ is given by the mass function

$$
\Phi(\underline{P})=(0.31983,0.163166,0.14233,0.17233,0.20233) .
$$

This does not belong to $\mathcal{M}(\underline{P})$ because $\Phi(\underline{P})(\{1,4\})=0.49216<0.5=\underline{P}(\{1,4\})$. On the other hand, the normalized Banzhaf value of $\underline{P}$ is given by:

$$
\Psi(\underline{P})=\left(\frac{5.25}{15.79}, \frac{2.49}{15.79}, \frac{2.07}{15.79}, \frac{2.75}{15.79}, \frac{3.23}{15.79}\right) .
$$

\footnotetext{
$\mathrm{d} \mathcal{F}$ is a filter of subsets of $\Omega$ is it is non-empty, for every $A, B \in \mathcal{F}$ there exists $C \in \mathcal{F}$ such that $C \subseteq A$ and $C \subseteq B$, and for every $A \in \mathcal{F}$ and $B$ such that $A \subseteq B$, then $B \in \mathcal{F}$.
} 
It then holds that, for $A=\{2,3,5\}, \Psi(\underline{P}(A))=\frac{7.79}{15.79}<0.5=\underline{P}(A)$. Thus, $\Psi(\underline{P})$ does not belong to the core of $\underline{P}$.

Remark 2. Coherent lower probabilities are the weakest model we shall consider in the study we are carrying out in this paper; to see why, note that an even weaker model would be those lower probabilities that avoid sure $l o s s^{5}$, which are those for which the core $\mathcal{M}(\underline{P})$ is non-empty. They correspond to balanced games within game theory ${ }^{42}$. However, for them the Shapley and Banzhaf values need not belong to the core, not even for spaces of cardinality three, where coherence is undistinguishable from normalized 2 -monotonicity. This can be seen for instance with $\underline{P}$ given by

$$
\underline{P}(\{1\})=\underline{P}(\{2\})=\underline{P}(\{3\})=\underline{P}(\{2,3\})=0, \underline{P}(\{1,2\})=\underline{P}(\{1,3\})=1,
$$

and of course with $\underline{P}(\{1,2,3\})=1$. We obtain that the Shapley value is $\Phi(\underline{P})=$ $\left(\frac{2}{3}, \frac{1}{6}, \frac{1}{6}\right)$ and the normalized Banzhaf value is $\Psi(\underline{P})=\left(\frac{3}{5}, \frac{1}{5}, \frac{1}{5}\right)$, while the only element of $\mathcal{M}(\underline{P})$ is given by the mass function $(1,0,0)$.

That is why we are focusing on lower probabilities that satisfy (at least) the property of coherence.

In spite of these negative results, we shall prove that the Shapley and Banzhaf values belong to the core in a number of particular cases, and therefore in those instances it makes sense their use as probability transformations. We begin by considering the case of coherent lower probabilities that are the lower envelope of two probability measures. They may arise for instance when we are aggregating information from two different sources.

\subsection{Lower envelopes of two probability measures}

Proposition 12. Consider two probability measures $P_{1}, P_{2}$ on $\mathcal{P}(\Omega)$ and let $\underline{P}$ be the coherent lower probability they determine. Then $\Psi(\underline{P})(i)=\Phi(\underline{P})(i)=$ $\frac{P_{1}(\{i\})+P_{2}(\{i\})}{2}$ for every $i \in \Omega$.

Proof. Let us denote by $\bar{P}$ the conjugate of $\underline{P}$. Then $\underline{P}(A)+\bar{P}(A)=P_{1}(A)+P_{2}(A)$ for every $A \subseteq \Omega$.

We begin by establishing the result for the Shapley value. From Eq. (3), and 
recalling that we denote $t=|A|$,

$$
\begin{aligned}
& \Phi(\underline{P})(i)=\sum_{A \nsupseteq\{i\}} \frac{t !(n-t-1) !}{n !}(\underline{P}(A \cup\{i\})-\underline{P}(A)) \\
& =\frac{(n-1) !}{n !}(\underline{P}(\{i\})+\bar{P}(\{i\}))+\sum_{t=1}^{n-2} \sum_{A \nsupseteq\{i\},|A|=t} \frac{t !(n-t-1) !}{n !}(\underline{P}(A \cup\{i\})-\underline{P}(A)) \\
& =\frac{(n-1) !}{n !}(\underline{P}(\{i\})+\bar{P}(\{i\})) \\
& \quad+\sum_{t=1}^{n-2} \sum_{A \nsupseteq\{i\},|A|=t} \frac{t !(n-t-1) !}{n !}(1-\bar{P}(\Omega \backslash(A \cup\{i\}))-\underline{P}(A)) \\
& =\frac{(n-1) !}{n !}(\underline{P}(\{i\})+\bar{P}(\{i\}))+\sum_{t=1}^{n-2} \sum_{A \nsupseteq\{i\},|A|=t} \frac{t !(n-t-1) !}{n !}(1-\bar{P}(A)-\underline{P}(A)),
\end{aligned}
$$

where last equality holds because given $A$ with cardinality $t$, the set $\Omega \backslash(A \cup\{i\})$ has cardinality $(n-t-1)$.

Now, for any fixed $t \in\{1, \ldots, n-2\}$, it holds that

$$
\begin{aligned}
& \sum_{A \supseteq\{i\},|A|=t}(\bar{P}(A)+\underline{P}(A))=\sum_{A \nsupseteq\{i\},|A|=t}\left(P_{1}(A)+P_{2}(A)\right) \\
= & \left(\begin{array}{c}
n-2 \\
t-1
\end{array}\right)\left(2-P_{1}(\{i\})-P_{2}(\{i\})\right)=\left(\begin{array}{c}
n-2 \\
t-1
\end{array}\right)(2-\underline{P}(\{i\})-\bar{P}(\{i\})),
\end{aligned}
$$

taking into account that any $j \neq i$ can be included in $\left(\begin{array}{c}n-2 \\ t-1\end{array}\right)$ different subsets of $\Omega \backslash\{i\}$ of cardinality $t$.

Thus, Eq. (20) is equal to

$$
\begin{aligned}
(\underline{P}(\{i\})+\bar{P}(\{i\}))\left[\frac{(n-1) !}{n !}+\sum_{t=1}^{n-2} \frac{t !(n-t-1) !}{n !}\left(\begin{array}{c}
n-2 \\
t-1
\end{array}\right)\right] & \\
& +\sum_{t=1}^{n-2} \frac{t !(n-t-1) !}{n !}\left[\left(\begin{array}{c}
n-1 \\
t
\end{array}\right)-2\left(\begin{array}{c}
n-2 \\
t-1
\end{array}\right)\right] .
\end{aligned}
$$

The first term is equal to

$$
\begin{aligned}
(\underline{P}(\{i\})+ & \bar{P}(\{i\}))\left[\frac{1}{n}+\sum_{t=1}^{n-2} \frac{t !(n-t-1) !}{n !} \frac{(n-2) !}{(t-1) !(n-t-1) !}\right] \\
& =(\underline{P}(\{i\})+\bar{P}(\{i\}))\left[\frac{1}{n}+\sum_{t=1}^{n-2} \frac{t}{n(n-1)}\right] \\
& =(\underline{P}(\{i\})+\bar{P}(\{i\}))\left[\frac{1}{n}+\frac{n-2}{2 n}\right]=\frac{\frac{P}{(\{i\})+\bar{P}(\{i\})}}{2},
\end{aligned}
$$


while the second term is equal to

$$
\begin{aligned}
& \sum_{t=1}^{n-2} \frac{t !(n-t-1) !}{n !}\left(\begin{array}{c}
n-1 \\
t
\end{array}\right)-2 \sum_{t=1}^{n-2} \frac{t !(n-t-1) !}{n !}\left(\begin{array}{c}
n-2 \\
t-1
\end{array}\right) \\
& =\sum_{t=1}^{n-2} \frac{1}{n}-2 \sum_{t=1}^{n-2} \frac{t}{n(n-1)}=\frac{n-2}{n}-\frac{n-2}{n}=0 .
\end{aligned}
$$

We conclude that $\Phi(\underline{P})(i)=\frac{P(\{i\})+\bar{P}(\{i\})}{2}=\frac{P_{1}(\{i\})+P_{2}(\{i\})}{2}$.

Consider next the Banzhaf value. Reasoning as before, we obtain

$$
B(\underline{P})(i)=\frac{1}{2^{n-1}}(\underline{P}(\{i\})+\bar{P}(\{i\}))+\sum_{t=1}^{n-2} \sum_{A \nsupseteq\{i\},|A|=t} \frac{1}{2^{n-1}}(1-\bar{P}(A)-\underline{P}(A)),
$$

which in turn, using Eq. (21), is equal to

$$
(\underline{P}(\{i\})+\bar{P}(\{i\}))\left[\frac{1}{2^{n-1}}+\sum_{t=1}^{n-2} \frac{1}{2^{n-1}}\left(\begin{array}{c}
n-2 \\
t-1
\end{array}\right)\right]+\sum_{t=1}^{n-2} \frac{1}{2^{n-1}}\left[\left(\begin{array}{c}
n-1 \\
t
\end{array}\right)-2\left(\begin{array}{c}
n-2 \\
t-1
\end{array}\right)\right] .
$$

Now, $\sum_{t=1}^{n-2}\left(\begin{array}{c}n-1 \\ t\end{array}\right)=2^{n-1}-2=2 \cdot\left(2^{n-2}-1\right)=2 \sum_{t=1}^{n-2}\left(\begin{array}{c}n-2 \\ t-1\end{array}\right)$, whence the last term is equal to 0 . Moreover,

$$
\frac{1}{2^{n-1}}+\sum_{t=1}^{n-2} \frac{1}{2^{n-1}}\left(\begin{array}{c}
n-2 \\
t-1
\end{array}\right)=\frac{1}{2^{n-1}}\left(1+2^{n-2}-1\right)=\frac{1}{2} .
$$

Thus, $B(\underline{P})(i)=\frac{P_{1}(\{i\})+P_{2}(\{i\})}{2}=\Phi(\underline{P})(i)$. From this it follows that the Banzhaf value is normalized:

$$
\sum_{i \in \Omega} B(\underline{P})(i)=\sum_{i \in \Omega} \frac{P_{1}(\{i\})+P_{2}(\{i\})}{2}=1,
$$

and as a consequence $B(\underline{P})(i)=\Psi(\underline{P})(i)=\Phi(\underline{P})(i)$ for every $i \in \Omega$.

\subsection{Four-element space}

Interestingly, in the case considered in the proposition above the Banzhaf value is always normalized. On the other hand, the result does not extend to coherent lower probabilities that are the envelope of three probability measures, as Example 4 shows.

Another situation in which we can guarantee that the Shapley value of a coherent lower probability belongs to its core is when the possibility space has cardinality four, as our next result shows:

Proposition 13. Let $\Omega=\{1,2,3,4\}$ and let $\underline{P}: \mathcal{P}(\Omega) \rightarrow[0,1]$ be a coherent lower probability. Then, $\Phi(\underline{P})$ belongs to $\mathcal{M}(\underline{P})$. 
Proof. Let us prove that $\Phi(\underline{P})(A) \geq \underline{P}(A)$ for every $A \subseteq \Omega$. Consider first of all $A=\{i\}$. From Eq. (3),

$$
\Phi(\underline{P})(\{i\})=\sum_{B \nsupseteq\{i\}} \frac{t !(n-t-1) !}{n !}(\underline{P}(B \cup\{i\})-\underline{P}(B)) \in[\underline{P}(\{i\}), \bar{P}(\{i\})],
$$

taking into account that, since $\underline{P}$ is coherent,

$$
\underline{P}(\{i\})+\underline{P}(B) \leq \underline{P}(B \cup\{i\}) \leq \underline{P}(B)+\bar{P}(\{i\})
$$

for every $B$ that does not include $i$. We deduce that $\Phi(\underline{P})(A) \geq \underline{P}(A)$ for any set $A$ with $|A|=1,3$. It remains thus to prove that $\Phi(\underline{P})(A) \geq \underline{P}(A)$ when $|A|=2$. Take for instance $A=\{1,2\}$; the proof the remaining sets is analogous.

Applying Eq. (3), we obtain that $\Phi(\underline{P})(\{1,2\})=\Phi(\underline{P})(\{1\})+\Phi(\underline{P})(\{2\})$ is equal to

$$
\begin{aligned}
& \frac{12}{24}+\frac{4}{24}[\underline{P}(\{1\})-\underline{P}(\{2,3,4\})+\underline{P}(\{2\})-\underline{P}(\{1,3,4\}) \\
& +\underline{P}(\{1,2\})-\underline{P}(\{3\})-\underline{P}(\{4\}+\underline{P}(\{1,2,3\})+\underline{P}(\{1,2,4\})-\underline{P}(\{3,4\})],
\end{aligned}
$$

whence it dominates $\underline{P}(\{1,2\})$ if and only if

$$
\begin{aligned}
\underline{P}(\{1\}) & +\underline{P}(\{2\})+\underline{P}(\{1,2,3\})+\underline{P}(\{1,2,4\})+3 \\
& \geq 5 \underline{P}(\{1,2\})+\underline{P}(\{3\})+\underline{P}(\{4\}+\underline{P}(\{2,3,4\})+\underline{P}(\{1,3,4\})+\underline{P}(\{3,4\}) .
\end{aligned}
$$

Now, as a consequence of the coherence of $\underline{P}$ we have the following inequalities (see ${ }^{5}$, Theorem 2.7.4):

$$
\begin{aligned}
\underline{P}(\{1,2\})+\underline{P}(\{2,3,4\}) & \leq 1+\underline{P}(\{2\}) \\
\underline{P}(\{1,2\})+\underline{P}(\{1,3,4\}) & \leq 1+\underline{P}(\{1\}) \\
\underline{P}(\{1,2\})+\underline{P}(\{3,4\}) & \leq 1 \\
\underline{P}(\{1,2\})+\underline{P}(\{3\}) & \leq \underline{P}(\{1,2,3\}) \\
\underline{P}(\{1,2\})+\underline{P}(\{4\}) & \leq \underline{P}(\{1,2,4\}) .
\end{aligned}
$$

Their addition implies that $\Phi(\underline{P})(\{1,2\}) \geq \underline{P}(\{1,2\})$. This conludes the proof.

Note that a similar result does not hold for the normalized Banzhaf value, as we can see from Example 3.

\subsection{Comparative lower probabilities}

Our attention shifts now to another useful model of non-additive measures: comparative probabilities. These ${ }^{36,37}$ correspond to the case where the available information about the probability of the events is of qualitative nature, in the sense that we can only make statements of the type "the probability of A is at least as much as that of B'. From the point of view of coalitional game theory, these models would appear if it is possible to give a partial order among the coalitions, so that all we 
can tell in some cases that the reward associated with the coalition of the players in $A$ is at least as large as the coalition of the players in $B$.

The mathematical study of comparative models can be complicated, in the sense that it is not guaranteed the existence of an additive model that is compatible with them ${ }^{38,39}$; we refer to ${ }^{40}$ for a survey of this topic. In ${ }^{41}$, the particular case of elementary comparative probabilities was considered, where we only give qualitative assessments about the value of individual players. This motivates the following definition:

Definition 5. Let $\Omega$ be a possibility space, and consider $\mathcal{I} \subseteq \Omega \times \Omega$. The (elementary) comparative model determined by $\mathcal{I}$ is the lower envelope $\underline{P}$ of the set

$$
\mathcal{M}:=\{P \text { probability measure }: P(\{i\}) \geq P(\{j\}) \forall(i, j) \in \mathcal{I}\} .
$$

It was proven in ${ }^{41}$ that the core of these models can be given quite a neat structure, and that it has at most $2^{n-1}$ different extreme points. However, the lower probability induced by this core need not be 2 -monotone in general (see ${ }^{41}$, Section 4.3). Taking this into account, it is not surprising to see that the pignistic transformation cannot be computed by means of Eq. (5), as our next example shows:

Example 5. Let us consider the comparative assessments

$$
P(\{1\}) \geq P(\{2\}), P(\{1\}) \geq P(\{3\}), P(\{2\}) \geq P(\{4\}), P(\{3\}) \geq P(\{4\}) .
$$

If we consider the set of probability measures compatible with these assessments, it follows from Theorem 1 in $^{41}$ that the extreme points of this set are the probability measures

$$
(1,0,0,0),\left(\frac{1}{2}, \frac{1}{2}, 0,0\right),\left(\frac{1}{2}, 0, \frac{1}{2}, 0\right),\left(\frac{1}{4}, \frac{1}{4}, \frac{1}{4}, \frac{1}{4}\right),\left(\frac{1}{3}, \frac{1}{3}, \frac{1}{3}, 0\right),
$$

From this we deduce that the lower probability $\underline{P}$ associated with these assessments is the lower envelope of these extreme points. It is given by $\underline{P}(A)=0$ if $1 \notin A$, and

$$
\begin{aligned}
& \underline{P}(\{1\})=\frac{1}{4}, \underline{P}(\{1,2\})=\underline{P}(\{1,3\})=\frac{1}{2}, \underline{P}(\{1,4\})=\frac{1}{3}, \\
& \underline{P}(\{1,2,3\})=\frac{3}{4}, \underline{P}(\{1,2,4\})=\underline{P}(\{1,3,4\})=\frac{1}{2}, \underline{P}(\Omega)=1 .
\end{aligned}
$$

Now, from Eq. (3), the pignistic transformation is given by

$$
\Phi(\underline{P})=\left(\frac{41}{72}, \frac{13}{72}, \frac{13}{72}, \frac{5}{72}\right) .
$$

On the other hand, in this case there are permutations $\sigma$ whose associated probability by Eq. (4) does not belong to the core $\mathcal{M}(\underline{P})$ : if we take for instance $\sigma=(1,2,4,3)$, we obtain $P_{\sigma}=\left(\frac{1}{4}, \frac{1}{4}, \frac{1}{2}, 0\right)$, and $P_{\sigma}(\{1,4\})=\frac{1}{4}<\underline{P}(\{1,4\})$. If we apply Eq. (5), we obtain the probability measure

$$
\left(\frac{167}{288}, \frac{49}{288}, \frac{49}{288}, \frac{23}{288}\right)
$$


that belongs to $\mathcal{M}(\underline{P})$ but does not coincide with $\Phi(\underline{P})$.

Next we prove that both the Shapley and the normalized Banzhaf values belong to the core in this case:

Proposition 14. Let $\underline{P}$ be a lower probability determined by elementary comparative probabilities. Then $\Phi(\underline{P})$ and $\Psi(\underline{P})$ belong to the core $\mathcal{M}(\underline{P})$.

Proof. Consider $i, j \in\{1, \ldots, n\}$ such that the assessment $P(\{i\}) \geq P(\{j\})$ is one of the comparative probability assessments in the definition of $\underline{P}$. Then it follows from Eq. (3) that

$$
\begin{aligned}
& \Phi(\underline{P})(i)=\sum_{A \nsupseteq\{i\}} \frac{t !(n-t-1) !}{n !}(\underline{P}(A \cup\{i\})-\underline{P}(A)) \text { and } \\
& \Phi(\underline{P})(j)=\sum_{A \nsupseteq\{j\}} \frac{t !(n-t-1) !}{n !}(\underline{P}(A \cup\{j\})-\underline{P}(A)) .
\end{aligned}
$$

Consider a set $A \subseteq \Omega \backslash\{i\}$. There are two possibilities:

- If $A \subseteq \Omega \backslash\{j\}$, then $\underline{P}(A \cup\{i\})-\underline{P}(A) \geq \underline{P}(A \cup\{j\})-\underline{P}(A) \Longleftrightarrow \underline{P}(A \cup\{i\}) \geq$ $\underline{P}(A \cup\{j\})$. For every extreme point $P$ of $\mathcal{M}(\underline{P})$ it holds that $P(A \cup\{i\})-P(A \cup$ $\{j\})=P(\{i\})-P(\{j\}) \geq 0$, and since $\underline{P}$ is the lower envelope of $\mathcal{M}(\underline{P})$, we also conclude that $\underline{P}(A \cup\{i\}) \geq \underline{P}(A \cup\{j\})$, simply by considering the extreme point where the value $\underline{P}(A \cup\{i\})$ is attained.

- If $j \in A$, we consider $A^{\prime}:=A \cup\{i\} \backslash\{j\} \subseteq \Omega \backslash\{j\}$. Then $\underline{P}(A \cup\{i\})-\underline{P}(A) \geq$ $\underline{P}\left(A^{\prime} \cup\{j\}\right)-\underline{P}\left(A^{\prime}\right) \Longleftrightarrow \underline{P}\left(A^{\prime}\right) \geq \underline{P}(A) \Longleftrightarrow \underline{P}(A \cup\{i\} \backslash\{j\}) \geq \underline{P}(A)$. Again, for every extreme point $P$ of $\mathcal{M}(\underline{P})$ it holds that $P(A \cup\{i\} \backslash\{j\})-P(A)=$ $P(\{i\})-P(\{j\}) \geq 0$, and since $\underline{P}$ is the lower envelope of $\mathcal{M}(\underline{P})$, we also conclude that $\underline{P}(A \cup\{i\} \backslash\{j\}) \geq \underline{P}(A)$, by considering the extreme point where $\underline{P}(A \cup\{i\} \backslash\{j\})$ is attained.

If we now consider that in the above procedure we are making a one-to-one correspondence between the families $\{A \subseteq \Omega \backslash\{i\}\}$ and $\{A \subseteq \Omega \backslash\{j\}\}$, we conclude that $\Phi(\underline{P})(i) \geq \Phi(\underline{P})(j)$. Since this holds for any of the elementary comparative assessments in the definition of $\underline{P}$, we conclude that $\Phi(\underline{P})$ belongs to the core $\mathcal{M}(\underline{P})$.

The proof of the inclusion of $\Psi(\underline{P})$ in the core is similar: by Eq. (8),

$$
\begin{aligned}
& \Psi(\underline{P})(i)=\sum_{A \nsupseteq\{i\}} \frac{1}{2^{n-1}}(\underline{P}(A \cup\{i\})-\underline{P}(A)) \text { and } \\
& \Psi(\underline{P})(j)=\sum_{A \nsupseteq\{j\}} \frac{1}{2^{n-1}}(\underline{P}(A \cup\{j\})-\underline{P}(A)) .
\end{aligned}
$$

If we notice that the weights $\frac{t !(n-t-1) !}{n !}$ played no role in the proof above for the Shapley value, we conclude that $\Psi(\underline{P})(i) \geq \Psi(\underline{P})(j)$ and therefore $\Psi(\underline{P})$ belongs to $\mathcal{M}(\underline{P})$. 


\section{Conclusions}

The results in this paper show that some of the nice properties of the Shapley value can be extended beyond the framework of 2-monotone lower probabilities and belief functions. We have considered a number of imprecise probability models; the relationship between them is summarised by the following figure, where the arrow means an inclusion between the two families:

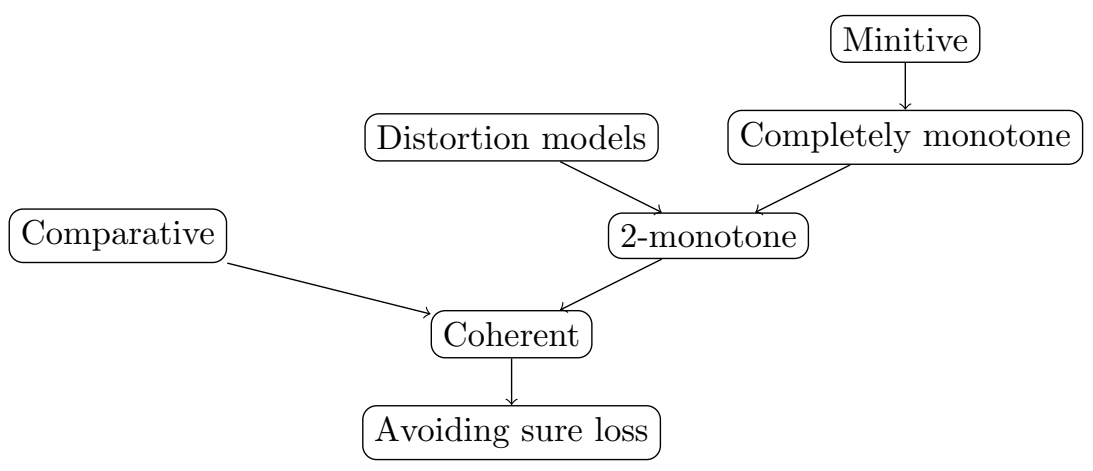

With respect to the Banzhaf value, although the lack of efficiency leads to the definition of the normalized version, it is also possible to prove its consistency with the core in a number of cases. Our results are summarised in the following table:

\begin{tabular}{c|c|c|c|c}
$\begin{array}{c}\text { Properties } \\
\text { of } \underline{P}\end{array}$ & $\begin{array}{c}\Phi(\underline{P}) \\
\text { in core? }\end{array}$ & $\begin{array}{c}\Psi(\underline{P}) \\
\text { in core? }\end{array}$ & $\begin{array}{c}\Phi(\underline{P}) \text { center } \\
\text { of gravity? }\end{array}$ & $\sum_{i} B(\underline{P})(i) \leq 1 ?$ \\
\hline Coherent, $|\Omega| \leq 3$ & YES & YES & YES & NO \\
Coherent, $|\Omega| \leq 4$ & YES & NO & NO & NO \\
Comparative & YES & YES & NO & NO \\
Coherent & NO & NO & NO & NO \\
Distortion & YES & YES & YES & NO \\
2-monotone & YES & NO & YES & NO \\
Probability intervals & YES & NO & YES & NO \\
Belief & YES & NO & YES & YES \\
Minitive & YES & YES & YES & YES
\end{tabular}

Although in this paper we have focused on the consistency with these game values with the core, in the future we should deepen into the investigation of the mathematical properties of these models as probability transformations, so as to be able to compare them properly with the existing models. In addition, it may be interesting to extend the study to other relevant models within imprecise probability theory, such as probability intervals ${ }^{28}$ or probability boxes ${ }^{43}$.

More generally, we would like to continue this research by considering the probabilistic solutions of games considered in ${ }^{15}$. In addition, we should also study the properties of other probability transformations, such as the maximum entropy one, for some of the imprecise probability models considered in this paper. 


\section{Acknowledgements}

This is an updated version, with proofs and additional results, of a paper presented at ISIPTA'2017 ${ }^{44}$. We acknowledge the financial support by project TIN201459543-P. We would also like to thank Paolo Vicig and the anonymous reviewers for some helpful comments.

\section{References}

1. M. Grabisch, Set functions, games and capacities in decision making Springer, 2016.

2. G. Choquet, "Theory of capacities", Annales de l'Institut Fourier 5 (1954) 131-295.

3. G. Shafer, A Mathematical Theory of Evidence Princeton University Press, Princeton, NJ, 1976.

4. D. Dubois and H. Prade, "Possibility theory: qualitative and quantitative aspects", in Handbook on Defeasible Reasoning and Uncertainty Management Systems, Vol.1: Quantified Representation of Uncertainty and Imprecision, Ph. Smets editor, (Kluwer Academic Publishers, Dordrecht, 1998) pp. 169-226.

5. P. Walley, Statistical Reasoning with Imprecise Probabilities Chapman and Hall, London, 1991.

6. P. Baroni and P. Vicig, "An uncertainty interchange format with imprecise probabilities, International Journal of Approximate Reasoning 40 (2005) 147-180.

7. J.-Y. Jaffray, "On the maximum entropy probability which is consistent with a convex capacity", International Journal of Uncertainty, Fuzziness and Knowledge-Based Systems 3 (1995) 27-33.

8. P. Smets, "Decision making in the TBM: the necessity of the pignistic transformation", International Journal of Approximate Reasoning 38 (2005) 133-147.

9. F. Voorbraak, "A computationally efficient approximation of Dempster-Shafer theory", International Journal of Man-Machine Studies 30 (1989) 525-536.

10. D. Dubois and H.Prade, Fuzzy Sets and Systems. Theory and Applications Academic Press, New York, 1980.

11. P. Williams, "Discussion of 'Belief functions and parametric models', by G. Shafer", Journal of the Royal Statistical Society, Series B 44(3) (1982) 341-343.

12. L.S. Shapley, "A value for n-person games", Annals of Mathematical Studies 28 (1953) 307-317.

13. L.S. Shapely, "Cores of convex games", International Journal of Game Theory $\mathbf{1}$ (1971) 11-26.

14. D. Denneberg, Non-Additive Measure and Integral Kluwer Academic, Dordrecht, 1994.

15. R.J. Weber, "Probabilistic values for games", in The Shapley value. Essays in honour of L.S. Shapley, A.E. Roth editor, (Cambridge University Press, 1988) pp. 101-119.

16. A.P. Dempster, "Upper and lower probabilities induced by a multivalued mapping", Annals of Mathematical Statistics 38 (1967) 325-339.

17. D. Dubois and H. Prade, "On several representations of an uncertain body of evidence", in Fuzzy Information and Decision Processes, M. Gupta and E. Sánchez editors (North Holland, 1982), pp. 167-181.

18. P. Smets and R. Kennes, "The transferable belief model", Artificial Intelligence $\mathbf{6 6 ( 2 )}$ (1994) 191-234.

19. A. Aregui and T. Denouex, "Constructing consonant belief functions from sample data using confidence sets of pignistic probabilities", International Journal of Approximate Reasoning 49 (2008) 575-594.

20. P. Monney, M. Chan and P. Romberg, "A belief function classifier based on informa- 
tion provided by noisy and dependent features", International Journal of Approximate Reasoning 52(3) (2011) 335-352.

21. J.F. Banzhaf, "Weighted voting does not work: a mathematical analysis", Rutgers Law Review 19 (1965) 317-343.

22. G. Owen, "A characterization of the Banzhaf-Coleman index", SIAM Journal of Applied Mathematics 35 (1978) 345-327.

23. R. Van der Brink and G. Van der Laan, "Axiomatizations of the normalized Banzhaf value and the Shapley value", Social Choice and Welfare 15 567-582.

24. P. Dubey and L.S. Shapely, "Mathematical properties of the Banzhaf power index", Mathematics and Operations Research 4 (1979) 99-131.

25. H.T. Nguyen, N.T. Nguyen and T. Wang, "On capacity functionals in interval probabilities", International Journal of Uncertainty, Fuzziness and Knowledge-Based Systems 5 (1997) 359-377.

26. D. Dubois and H. Prade, "Unfair coins and necessity measures: towards a possibilistic interpretation of histograms", Fuzzy Sets and Systems 10 (1983) 15-20.

27. P. Walley, Coherent lower (and upper) probabilities, Statistics Research Report 22, University of Warwick, Coventry, 1981.

28. L.M. de Campos, J.F. Huete and S. Moral, "Probability intervals: a tool for uncertain reasoning", International Journal of Uncertainty, Fuzziness and Knowledge-Based Systems 2 (1994) 167-196.

29. I. Montes, E. Miranda and S. Destercke, "Pari-Mutuel probabilities as an uncertainty model", Submitted for publication (2017).

30. I. Montes, E. Miranda and S. Destercke, "A study of the Pari-Mutuel Model from the point of view of Imprecise Probabilities", Proc. 10th International Symposium on Imprecise Probability: Theories and Applications, ISIPTA 2017, Lugano, Switzerland, 2017, pp. 229-240.

31. R. Pelessoni, P. Vicig and M. Zaffalon, "Inference and risk measurement with the pari-mutuel model", International Journal of Approximate Reasoning 51(9) (2010) $1145-1158$.

32. G. de Cooman, M.C.M. Troffaes and E. Miranda, " $n$-Monotone exact functionals", Journal of Mathematical Analysis and Applications 347 (2008) 143-156.

33. L. Utkin, "A framework for imprecise robust one-class classification models", Journal of Machine Learning Research and Cybernetics 5(3) (2014) 379-393.

34. L. Utkin and A. Wiencierz, "An imprecise boosting-like approach to regression", in Proc. of the 8th International Symposium on Imprecise Probability: Theory and Applications, 2013, pp. 345-354.

35. G. Owen, Game theory Academic Press, San Diego, Third Edition, 1995.

36. B. de Finetti, "Sul significato soggettivo della probabilità", Fundamenta Mathematicae 17 (1931) 298-329.

37. B. Koopman, "The bases of probability", Bulletin of the Ameraican Mathematical Society 46 (1940) 763-774.

38. M. Kaplan and T. Fine, "Joint orders in comparative probability", Annals of Probability 5 (1977) 161-179.

39. C. Kraft, J. Pratt and A. Seidenberg, "Intuitive probability on finite sets", The Annals of Mathematical Statistics 30 408-419.

40. C. Regoli, "Comparative probability and robustness", Lecture Notes-Monograph Series 29 (1996) 343-352.

41. E. Miranda and S. Destercke, "Extreme points of the credal sets generated by comparative probabilities", Journal of Mathematical Psychology 64/65 (2015) 44-57.

42. L.S. Shapley, "On balanced sets and cores", Naval Research Logistic Quarterly 14 
(1967) 453-460.

43. S. Ferson, V. Kreinovich, L. Ginzburg, D.S. Myers and K. Sentz, Constructing probability boxes and Dempster-Shafer structures Technical report SAND2002-4015, Sandia National Laboratories, 2003.

44. E. Miranda and I. Montes, "Game solutions, probability transformations and the core", Proc. 10th International Symposium on Imprecise Probability: Theories and Applications, ISIPTA 2017, Lugano, Switzerland, 2017, pp. 217-228. 